\title{
Plant Preparations and Compounds with Activities against Biofilms Formed by Candida spp.
}

\author{
Tomasz M. Karpiński ${ }^{1, *(D)}$, Marcin Ożarowski ${ }^{2}$ (D), Agnieszka Seremak-Mrozikiewicz ${ }^{3,4,5}$, Hubert Wolski $^{3,6}$ \\ and Artur Adamczak 7 (D)
}

1 Department of Medical Microbiology, Poznań University of Medical Sciences, Wieniawskiego 3, 61-712 Poznań, Poland

2 Department of Biotechnology, Institute of Natural Fibres and Medicinal Plants, National Research Institute, Wojska Polskiego 71b, 60-630 Poznań, Poland; marcin.ozarowski@iwnirz.pl

3 Division of Perinatology and Women's Diseases, Poznań University of Medical Sciences, Polna 33, 60-535 Poznań, Poland; asm@data.pl (A.S.-M.); hubertwolski@wp.pl (H.W.)

4 Laboratory of Molecular Biology in Division of Perinatology and Women's Diseases, Poznań University of Medical Sciences, Polna 33, 60-535 Poznań, Poland

5 Department of Pharmacology and Phytochemistry, Institute of Natural Fibres and Medicinal Plants, National Research Institute, Kolejowa 2, 62-064 Plewiska, Poland

6 Division of Gynecology and Obstetrics, Podhale Multidisciplinary Hospital, Szpitalna 14, 34-400 Nowy Targ, Poland

7 Department of Botany, Breeding and Agricultural Technology of Medicinal Plants, Institute of Natural Fibres and Medicinal Plants, National Research Institute, Kolejowa 2, 62-064 Plewiska, Poland; artur.adamczak@iwnirz.pl

check for updates

Citation: Karpiński, T.M.;

Ożarowski, M.;

Seremak-Mrozikiewicz, A.; Wolski,

H.; Adamczak, A. Plant Preparations and Compounds with Activities against Biofilms Formed by Candida spp. J. Fungi 2021, 7, 360. https:// doi.org/10.3390/jof7050360

Academic Editors: Célia F. Rodrigues and Jesus A. Romo

Received: 20 March 2021

Accepted: 1 May 2021

Published: 5 May 2021

Publisher's Note: MDPI stays neutral with regard to jurisdictional claims in published maps and institutional affiliations.

Copyright: (c) 2021 by the authors. Licensee MDPI, Basel, Switzerland. This article is an open access article distributed under the terms and conditions of the Creative Commons Attribution (CC BY) license (https:// creativecommons.org/licenses/by/ $4.0 /)$.
* Correspondence: tkarpin@ump.edu.pl; Tel.: +48-61-854-61-38

\begin{abstract}
Fungi from the genus Candida are very important human and animal pathogens. Many strains can produce biofilms, which inhibit the activity of antifungal drugs and increase the tolerance or resistance to them as well. Clinically, this process leads to persistent infections and increased mortality. Today, many Candida species are resistant to drugs, including C. auris, which is a multiresistant pathogen. Natural compounds may potentially be used to combat multiresistant and biofilm-forming strains. The aim of this review was to present plant-derived preparations and compounds that inhibit Candida biofilm formation by at least 50\%. A total of 29 essential oils and 16 plant extracts demonstrate activity against Candida biofilms, with the following families predominating: Lamiaceae, Myrtaceae, Asteraceae, Fabaceae, and Apiacae. Lavandula dentata (0.045-0.07 mg/L), Satureja macrosiphon $(0.06-8 \mathrm{mg} / \mathrm{L})$, and Ziziphora tenuior $(2.5 \mathrm{mg} / \mathrm{L})$ have the best antifungal activity. High efficacy has also been observed with Artemisia judaica, Lawsonia inermis, and Thymus vulgaris. Moreover, 69 plant compounds demonstrate activity against Candida biofilms. Activity in concentrations below $16 \mathrm{mg} / \mathrm{L}$ was observed with phenolic compounds (thymol, pterostilbene, and eugenol), sesquiterpene derivatives (warburganal, polygodial, and ivalin), chalconoid (lichochalcone A), steroidal saponin (dioscin), flavonoid (baicalein), alkaloids (waltheriones), macrocyclic bisbibenzyl (riccardin D), and cannabinoid (cannabidiol). The above compounds act on biofilm formation and/or mature biofilms. In summary, plant preparations and compounds exhibit anti-biofilm activity against Candida. Given this, they may be a promising alternative to antifungal drugs.
\end{abstract}

Keywords: Candida; biofilm; treatment; antifungals; natural compounds; essential oil; extract; minimal inhibitory concentration (MIC)

\section{Introduction}

The genus Candida contains about 150 species; however, most are environmental organisms. The most medically important is Candida albicans, which accounts for about $80 \%$ of infections. C. albicans causes more than 400,000 cases of bloodstream life-threatening infections annually, with a mortality rate of about $42 \%$ [1]. Candida non-albicans species that 
are mainly responsible for infections are C. glabrata, C. parapsilosis, C. tropicalis, C. krusei, and C. dubliniensis [2]. Less frequently identified are C. guilliermondii, C. lusitaniae, C. rugosa, C. orthopsilosis, C. metapsilosis, C. famata, C. inconspicua, and C. kefyr [3].

C. albicans is a member of the commensal microflora. It colonizes the oral mucosal surface of $30-50 \%$ of healthy people. The rate of carriage increases with age and in persons with dental prostheses up to 60\% [4-6]. Opportunistic infection caused by Candida species is termed candidiasis. At least one episode of vulvovaginal candidiasis (or thrush) concerns 50 to $75 \%$ of women of childbearing age [7]. Candidiasis can also affect the oral cavity, penis, skin, nails, cornea, and other parts of the body. In immunocompromised persons, untreated candidiasis poses the risk of systemic infection and fungemia [5,8]. Candida can be an important etiological factor in the infection of chronic wounds that are difficult to treat; this is mainly related to the production of biofilm [9].

Treatment of candidiasis depends on the infection site and the patient's condition. According to guidelines, vulvovaginal candidiasis should be treated with oral or topical fluconazole; however, regarding C. glabrata infection, topical boric acid, nystatin, or flucytosine is suggested. In oropharyngeal candidiasis, the treatment options include clotrimazole, miconazole, or nystatin, and in severe disease, fluconazole or voriconazole. In candidemia and invasive candidiasis, the drugs of choice are echinocandins (caspofungin, micafungin, anidulafungin), fluconazole, or voriconazole; in resistant strains, amphoteticin B is used. In selected cases of candidemia caused by C. krusei, voriconazole is recommended [10-12]. More details can be found in the Guidelines of the Infectious Diseases Society of America [12] and the European Society of Clinical Microbiology and Infectious Diseases [11]. Increasingly, Candida species are becoming resistant to drugs. Marak and Dhanashree [13] tested the resistance of 90 Candida strains isolated from different clinical samples, such as pus, urine, blood, and body fluid. Their study revealed that about $41 \%$ of $C$. albicans strains are resistant to fluconazole and voriconazole. Simultaneously, about $41 \%$ of $C$. tropicalis strains are resistant to voriconazole and about $36 \%$ of strains to fluconazole. In strains of C. krusei, about $23 \%$ are resistant to fluconazole and about $18 \%$ to voriconazole. Rudramurthy et al. [14] studied resistance in C. auris, which is considered a multiresistant pathogen. Among 74 strains obtained from patients with candidemia, over $90 \%$ of strains were resistant to fluconazole and about $73 \%$ to voriconazole. Virulence factors of Candida species include the secretion of hydrolases, the transition of yeast to hyphae, phenotypic switching, and biofilm formation [15,16]. All microorganisms in biofilm form are more resistant to antimicrobial and host factors, which leads to difficulties in eradication [17]. It has also been shown that resistance to drugs increases significantly in the case of Candida biofilm occurrence. Biofilm prevents the spread of antifungals; moreover, fluconazole is bound by the biofilm matrix [18]. The formation of a Candida biofilm during infection increases mortality, length of hospital stay, and cost of antifungal therapy [19].

Due to the above, new antifungal drugs are sought that could effectively combat not only planktonic fungi but also fungal biofilms. The natural compounds offer promise, with many acting on Candida species or biofilms in vitro [20].

The aim of this review was to present plant-derived natural compounds that have an effect against biofilms formed by Candida species.

\section{Materials and Methods}

In this review, publications available in PubMed and Scopus databases and through the Google search engine were taken into account. The following keywords and their combinations were used: "antifungal," "Candida," "anti-biofilm," "biofilm," "plant," "compound," "extract," and "essential oil." The principal inclusion criterion was the inhibition of biofilm formation by at least $50 \%$. We focused on biofilm inhibition assays, in which the time of culture allowed for Candida biofilm maturation was at least 24 hours. Articles from the year 2000 to the present were taken into account. All articles published in predatory journals were rejected. 


\section{Results and Discussion}

\subsection{Plant Preparations That Display Activity against Candida Biofilms}

The present review includes 60 articles in which Candida biofilm formation was inhibited by at least $50 \%$. It has been shown that preparations from 34 plants demonstrate activity against Candida biofilms. Among them were 29 essential oils and 16 extracts. The plants from the following families dominated: Lamiaceae (6 species in 5 genera), Myrtaceae ( 5 species in 4 genera), Asteraceae (4 species in 4 genera), Fabaceae (4 species in 3 genera), and Apiacae (4 species in 2 genera).

Plants from the Lamiaceae family had the best antifungal activity, including Lavandula dentata $(0.045-0.07 \mathrm{mg} / \mathrm{L})$ [21], Satureja macrosiphon (0.06-8 mg/L) [22], and Ziziphora tenuior $(2.5 \mathrm{mg} / \mathrm{L})$ [23]. Artemisia judaica $(2.5 \mathrm{mg} / \mathrm{L})$ from the Asteraceae family [24], Lawsonia inermis (2.5-12.5 mg/L) from the Lythraceae family [25], and Thymus vulgaris (12.5 mg/L) from the Lamiaceae family [26] likewise exhibited good antifungal activity (Table 1). All preparations were essential oils, with the exception of Lawsonia inermis, which was an extract. Most of the plant preparations presented in Table 1 acted on biofilm formation and/or mature biofilms.

Table 1. Antifungal (MICs) and anti-biofilm (inhibition $>50 \%$ ) activity of plant preparations (essential oils or extracts).

\begin{tabular}{|c|c|c|c|c|c|c|}
\hline $\begin{array}{l}\text { Name of Plant } \\
\text { (Family) }\end{array}$ & $\begin{array}{l}\text { Main Compounds Presented } \\
\text { in the Reference } \\
\text { (EO: Essential Oil) }\end{array}$ & $\begin{array}{l}\text { Targeted } \\
\text { Species of } \\
\text { Candida }\end{array}$ & $\begin{array}{c}\mathrm{MICs} \\
(\mathrm{mg} / \mathrm{L} ; \mathrm{mL} / \mathrm{L})\end{array}$ & $\begin{array}{c}\text { Inhibition of Biofilm } \\
\text { Formation by at } \\
\text { Least } 50 \%(\mathrm{mg} / \mathrm{L} ; \\
\mathrm{mL} / \mathrm{L})\end{array}$ & $\begin{array}{l}\text { Inhibited Stage of } \\
\text { Biofilm; Method of } \\
\text { Biofilm Detection }\end{array}$ & Ref. \\
\hline $\begin{array}{c}\text { Acorus calamus var. angustatus } \\
\text { Besser }=\text { A. tatarinowii Schott } \\
\text { (Acoraceae) }\end{array}$ & $\begin{array}{c}\text { EO: asaraldehyde, } 1-(2,4,5- \\
\text { trimethoxyphenyl)-1,2-propanediol, } \\
\alpha \text {-asarone, } \beta \text {-asarone, } \gamma \text {-asarone, } \\
\text { acotatarone } C\end{array}$ & C. albicans & 51.2 & $50-200$ & $\begin{array}{l}\text { Mature biofilm; crystal } \\
\text { violet and fluorescence } \\
\text { microscopy }\end{array}$ & [27] \\
\hline $\begin{array}{l}\text { Allium sativum } \mathrm{L} \text {. } \\
\text { (Amaryllidaceae) }\end{array}$ & Extract: allicin & C. albicans & 400 & 60 & Biofilm formation; XTT & [28] \\
\hline $\begin{array}{c}\text { Aloysia gratissima (Aff \& } \\
\text { Hook).Tr } \\
\text { (Verbenaceae) }\end{array}$ & $\begin{array}{c}\text { EO: E-pinocamphone }(16.07 \%), \\
\beta \text {-pinene }(12.01 \%), \text { guaiol }(8.53 \%), \\
\text { E-pinocarveol acetate }(8.19 \%)\end{array}$ & C. albicans & 15 & 500 & $\begin{array}{l}\text { Biofilm formation; } \\
\text { crystal violet }\end{array}$ & [29] \\
\hline \multirow{5}{*}{$\begin{array}{l}\text { Artemisia judaica L. } \\
\quad \text { (Asteraceae) }\end{array}$} & \multirow{5}{*}{$\begin{array}{c}\text { EO: piperitone }(30.4 \%) \text {, camphor } \\
(16.1 \%) \text {, ethyl cinnamate }(11.0 \%) \\
\text { chrysanthenone }(6.7 \%)\end{array}$} & C. albicans & 1.25 & 2.5 & \multirow{5}{*}{ Mature biofilm; XTT } & \multirow{5}{*}{ [24] } \\
\hline & & C. guillermondii & 1.25 & 2.5 & & \\
\hline & & C. krusei & 1.25 & 2.5 & & \\
\hline & & C. parapsilosis & 1.25 & 2.5 & & \\
\hline & & C. tropicalis & 1.25 & 2.5 & & \\
\hline $\begin{array}{l}\text { Buchenavia tomentosa Eichler } \\
\text { (Combretaceae) }\end{array}$ & $\begin{array}{l}\text { Extract: gallic acid, kaempferol, } \\
\text { epicatechin, ellagic acid, vitexin, } \\
\text { and corilagin }\end{array}$ & C. albicans & 625 & 312.5 & $\begin{array}{l}\text { Biofilm formation } \\
\text { and mature } \\
\text { biofilm; culture }\end{array}$ & [30] \\
\hline $\begin{array}{c}\text { Chamaecostus cuspidatus } \\
\text { (Nees \& Mart.) C.Specht \& } \\
\text { D.W.Stev. } \\
\text { (Costaceae) }\end{array}$ & $\begin{array}{l}\text { Extract: dioscin, } \\
\text { aferoside A, aferoside C }\end{array}$ & C. albicans & 250 & 15.62 & $\begin{array}{l}\text { Biofilm formation and } \\
\text { mature biofilm; MTT }\end{array}$ & [31] \\
\hline \multirow{3}{*}{$\begin{array}{c}\text { Cinnamomum verum J. Presl } \\
\text { (Lauraceae) }\end{array}$} & \multirow{3}{*}{$\begin{array}{l}\text { EO: eugenol }(77.22 \%) \text {, benzyl benzoate } \\
(4.53 \%) \text {, trans-caryophyllene }(3.39 \%), \\
\text { acetyl eugenol }(2.75 \%) \text {, linalool } 2.11 \%\end{array}$} & C. albicans & 1000 & 150 & \multirow{3}{*}{ Biofilm adhesion; XTT } & \multirow{3}{*}{ [32] } \\
\hline & & C. dubliniensis & 1000 & 200 & & \\
\hline & & C. tropicalis & 1000 & 350 & & \\
\hline \multirow{6}{*}{$\begin{array}{l}\text { Citrus limon (L.) Osbeck } \\
\text { (Rutaceae) }\end{array}$} & \multirow{6}{*}{$\begin{array}{c}\text { EO: limonene }(53.4 \%) \text {, neral }(11 \%), \\
\text { geraniol }(9 \%) \text {, trans-limonene oxide } \\
(7 \%) \text {, nerol }(6 \%)\end{array}$} & C. albicans & 500 & 2000 & \multirow{6}{*}{$\begin{array}{l}\text { Biofilm formation and } \\
\text { mature biofilm; XTT }\end{array}$} & \multirow{6}{*}{ [33] } \\
\hline & & C. glabrata & 250 & 1000 & & \\
\hline & & C. krusei & 500 & 125 & & \\
\hline & & C. orthopsilosis & 500 & 1000 & & \\
\hline & & C. parapsilosis & 500 & 2000 & & \\
\hline & & C. tropicalis & 250 & 2000 & & \\
\hline $\begin{array}{l}\text { Copaifera paupera } \\
\text { (Herzog) Dwyer } \\
\text { (Fabaceae) }\end{array}$ & $\begin{array}{c}\text { Extract: galloylquinic acids, quercetrin, } \\
\text { afzelin }\end{array}$ & C. glabrata & 5.89 & 46.87 & $\begin{array}{l}\text { Biofilm formation and } \\
\text { mature biofilm; XTT }\end{array}$ & [34] \\
\hline $\begin{array}{c}\text { Copaifera reticulata Ducke } \\
\text { (Fabaceae) }\end{array}$ & $\begin{array}{c}\text { Extract: galloylquinic acids, quercetrin, } \\
\text { afzelin }\end{array}$ & C. glabrata & 5.89 & 46.87 & $\begin{array}{l}\text { Biofilm formation and } \\
\text { mature biofilm; XTT }\end{array}$ & [34] \\
\hline \multirow{5}{*}{$\begin{array}{l}\text { Coriandrum sativum } \mathrm{L} . \\
\text { (Apiaceae) }\end{array}$} & $\begin{array}{c}\text { EO: 1-decanol (33.91\%), E-2-decen-1-ol } \\
\text { (23.59\%), 2-dodecen-1-ol (13.06\%), } \\
\text { E-2-tetradecen-1-ol (5.46\%) }\end{array}$ & C. albicans & 7 & 250 & $\begin{array}{l}\text { Biofilm formation; } \\
\text { crystal violet }\end{array}$ & [29] \\
\hline & \multirow{4}{*}{$\begin{array}{c}\text { EO: decanal }(19.09 \%), \text { trans-2-decenal } \\
\text { (17.54\%), 2-decen-1-ol }(12.33 \%) \\
\text { cyclodecane }(12.15 \%)\end{array}$} & C. albicans & 15.6 & $62.5-125$ & \multirow{4}{*}{$\begin{array}{l}\text { Biofilm adhesion; } \\
\text { crystal violet }\end{array}$} & \multirow{4}{*}{ [35] } \\
\hline & & C. dubliniensis & 31.2 & $62.5-125$ & & \\
\hline & & C. rugosa & 15.6 & 62.5 & & \\
\hline & & C. tropicalis & 31.2 & $31.25-250$ & & \\
\hline
\end{tabular}


Table 1. Cont

\begin{tabular}{|c|c|c|c|c|c|c|}
\hline $\begin{array}{l}\text { Name of Plant } \\
\text { (Family) }\end{array}$ & $\begin{array}{l}\text { Main Compounds Presented } \\
\text { in the Reference } \\
\text { (EO: Essential Oil) }\end{array}$ & $\begin{array}{l}\text { Targeted } \\
\text { Species of } \\
\text { Candida }\end{array}$ & $\begin{array}{c}\text { MICs } \\
(\mathrm{mg} / \mathrm{L} ; \mathrm{mL} / \mathrm{L})\end{array}$ & $\begin{array}{c}\text { Inhibition of Biofilm } \\
\text { Formation by at } \\
\text { Least } 50 \%(\mathrm{mg} / \mathrm{L} \\
\mathrm{mL} / \mathrm{L})\end{array}$ & $\begin{array}{l}\text { Inhibited Stage of } \\
\text { Biofilm; Method of } \\
\text { Biofilm Detection }\end{array}$ & Ref. \\
\hline $\begin{array}{l}\text { Croton eluteria (L.) W.Wright } \\
\text { (Euphorbiaceae) }\end{array}$ & $\begin{array}{c}\text { EO: } \alpha \text {-pinene }(29.37 \%), \beta \text {-pinene } \\
(19.35 \%), \text { camphene }(10.31 \%) \\
1,8 \text {-cineole }(9.68 \%)\end{array}$ & C. albicans & 4000 & $5-500$ & $\begin{array}{l}\text { Biofilm formation; } \\
\text { confocal laser } \\
\text { microscopy }\end{array}$ & [36] \\
\hline \multirow{6}{*}{$\begin{array}{l}\text { Cupressus sempervirens L. } \\
\text { (Cupressaceae) }\end{array}$} & \multirow{6}{*}{$\begin{array}{l}\text { EO: sabinene }(20.3 \%) \text {, citral }(20 \%) \text {, } \\
\text { terpinene-4-ol }(15.4 \%), \alpha \text {-pinene }(8 \%)\end{array}$} & C. albicans & 250 & 1000 & \multirow{6}{*}{$\begin{array}{l}\text { Biofilm formation and } \\
\text { mature biofilm; XTT }\end{array}$} & \multirow{6}{*}[33]{} \\
\hline & & C. glabrata & 31.25 & 250 & & \\
\hline & & C. krusei & 62.5 & 62.5 & & \\
\hline & & C. orthopsilosis & 31.25 & 125 & & \\
\hline & & C. parapsilosis & 62.5 & 500 & & \\
\hline & & C. tropicalis & 250 & 500 & & \\
\hline $\begin{array}{l}\text { Cymbopogon citratus } \\
\text { (DC.) Stapf } \\
\text { (Poaceae) }\end{array}$ & EO: no composition & C. albicans & $180-360$ & $22.5-180$ & Biofilm formation; XTT & [37] \\
\hline $\begin{array}{l}\text { Cymbopogon martini (Roxb.) } \\
\text { W.Watson } \\
\text { (Poaceae) }\end{array}$ & EO: no composition & C. albicans & 16,800 & 800 & Biofilm formation; XTT & {$[38]$} \\
\hline \multirow{3}{*}{$\begin{array}{l}\text { Cymbopogon nardus (L.) } \\
\text { Rendle } \\
\text { (Poaceae) }\end{array}$} & \multirow{3}{*}{$\begin{array}{c}\text { EO: citronellal (27.87\%), } \\
\text { geraniol (22.77\%), geranial (14.54\%), } \\
\text { citronellol }(11.85 \%) \text {, neral }(11.21 \%)\end{array}$} & C. albicans & 1000 & $2500-5000$ & \multirow{3}{*}{ Biofilm adhesion; XTT } & \multirow{3}{*}[39]{} \\
\hline & & C. krusei & $250-500$ & 2500 & & \\
\hline & & C. parapsilosis & 500-1000 & $5000-10,000$ & & \\
\hline $\begin{array}{l}\text { Cyperus articulatus L. } \\
\text { (Cyperaceae) }\end{array}$ & $\begin{array}{c}\text { EO: } \alpha \text {-pinene }(5.72 \%), \text { mustakone } \\
(5.66 \%), \alpha \text {-bulnesene }(5.02 \%) \\
\alpha \text {-copaene }(4.97 \%)\end{array}$ & C. albicans & 125 & 250 & $\begin{array}{l}\text { Biofilm formation; } \\
\text { crystal violet }\end{array}$ & [29] \\
\hline $\begin{array}{l}\text { Eucalyptus sp. } \\
\text { (Myrtaceae) }\end{array}$ & EO: no composition & C. albicans & 8 & 8 & $\begin{array}{l}\text { Mature biofilm; } \\
\text { luminescence }\end{array}$ & [40] \\
\hline \multirow{4}{*}{$\begin{array}{l}\text { Eucalyptus globulus Labill. } \\
\text { (Myrtaceae) }\end{array}$} & \multirow{3}{*}{$\begin{array}{c}\text { EO: } 1,8 \text {-cineole }(75.8 \%), \text { p-cymene } \\
(7.5 \%), \alpha \text {-pinene }(7.4 \%) \text {, limonene } \\
(6.4 \%)\end{array}$} & C. albicans & 219 & $11,250-22,500$ & \multirow{3}{*}{$\begin{array}{l}\text { Mature biofilm; atomic } \\
\text { force microscopy }\end{array}$} & \multirow{3}{*}[41]{} \\
\hline & & C. glabrata & 219 & $11,250-22,500$ & & \\
\hline & & C. tropicalis & 885 & $11,250-22,500$ & & \\
\hline & EO: no composition & C. albicans & 8400 & 500 & Biofilm formation; XTT & [38] \\
\hline $\begin{array}{l}\text { Eugenia brasiliensis Lam. } \\
\text { (Myrtaceae) }\end{array}$ & Extract: no composition & C. albicans & $15.62-31.25$ & 156 & $\begin{array}{l}\text { Mature biofilm; } \\
\text { scanning electron } \\
\text { microscopy }\end{array}$ & [42] \\
\hline $\begin{array}{c}\text { Eugenia leitonii Legrand nom. } \\
\text { inval. } \\
\text { (Myrtaceae) }\end{array}$ & Extract: no composition & C. albicans & $15.62-250$ & 156 & $\begin{array}{l}\text { Mature biofilm; } \\
\text { scanning electron } \\
\text { microscopy }\end{array}$ & [42] \\
\hline $\begin{array}{l}\text { Helichrysum italicum (Roth) } \\
\text { G.Don } \\
\text { (Asteraceae) }\end{array}$ & $\begin{array}{c}\text { EO: } \alpha \text {-pinene }(27.64 \%), \gamma \text {-elemene } \\
(23.84 \%), \beta \text {-caryophyllene }(13.05 \%), \\
\alpha \text {-longipinene }(11.25 \%)\end{array}$ & C. albicans & 6000 & $10-500$ & $\begin{array}{l}\text { Biofilm formation; } \\
\text { confocal laser } \\
\text { microscopy }\end{array}$ & [36] \\
\hline \multirow{2}{*}{$\begin{array}{l}\text { Laserpitium latifolium L. } \\
\text { (Apiaceae) }\end{array}$} & \multirow[t]{2}{*}{ Extract: laserpitine } & C. albicans & 1250 & 6300 & \multirow{2}{*}{$\begin{array}{l}\text { Mature biofilm; } \\
\text { luminescence }\end{array}$} & \multirow{2}{*}{43} \\
\hline & & C. krusei & 1250 & 6300 & & \\
\hline \multirow{2}{*}{$\begin{array}{l}\text { Laserpitium ochridanum } \\
\text { Micevski } \\
\text { (Apiaceae) }\end{array}$} & \multirow{2}{*}{$\begin{array}{l}\text { Extract: isomontanolide, } \\
\text { montanolide, tarolide }\end{array}$} & C. albicans & 5000 & 10,000 & \multirow{2}{*}{$\begin{array}{l}\text { Mature biofilm; } \\
\text { luminescence }\end{array}$} & \multirow[t]{2}{*}[43]{} \\
\hline & & C. krusei & 5000 & 10,000 & & \\
\hline \multirow{2}{*}{$\begin{array}{c}\text { Laserpitium zernyi Hayek }=\text { L. } \\
\text { siler subsp. zernyi (Hayek) } \\
\text { Tutin }\end{array}$} & \multirow{2}{*}{$\begin{array}{l}\text { Extract: isomontanolide, } \\
\text { montanolide, tarolide }\end{array}$} & C. albicans & 7500 & 15,000 & \multirow{2}{*}{$\begin{array}{l}\text { Mature biofilm; } \\
\text { luminescence }\end{array}$} & \multirow[t]{2}{*}{ [43] } \\
\hline & & C. krusei & 7500 & 37,500 & & \\
\hline $\begin{array}{l}\text { (Apiaceae) } \\
\text { Lavandula dentata } \mathrm{L} . \\
\text { (Lamiaceae) }\end{array}$ & $\begin{array}{c}\text { EO: eucalyptol (42.66\%), } \beta \text {-pinene } \\
(8.59 \%), \text { trans- } \alpha \text {-bisabolene }(6.34 \%), \\
\text { pinocarveol }(6.3 \%)\end{array}$ & C. albicans & $0.15-0.18$ & $0.045-0.07$ & Mature biofilm; XTT & [21] \\
\hline $\begin{array}{l}\text { Lawsonia inermis L. } \\
\text { (Lythraceae) }\end{array}$ & Extract: no composition & C. albicans & 10 & $2.5-12.5$ & Mature biofilm; MTT & [25] \\
\hline $\begin{array}{l}\text { Lippia sidoides Cham. } \\
\text { (Verbenaceae) }\end{array}$ & $\begin{array}{c}\text { EO: thymol (65.76\%), p-cymene } \\
(17.28 \%), \alpha \text {-caryophyllene }(10.46 \%) \\
\text { cyclohexanone }(6.5 \%)\end{array}$ & C. albicans & 250 & 500 & $\begin{array}{l}\text { Biofilm formation; } \\
\text { crystal violet }\end{array}$ & [29] \\
\hline \multirow{6}{*}{$\begin{array}{l}\text { Litsea cubeba (Lour.) Pers. } \\
\text { (Lauraceae) }\end{array}$} & \multirow{6}{*}{$\begin{array}{l}\text { EO: limonene }(37 \%), \text { neral }(31.4 \%) \\
\text { citral }(12 \%), \text { linalool }(4 \%)\end{array}$} & C. albicans & 500 & 2000 & & \\
\hline & & C. glabrata & 250 & 2000 & & \\
\hline & & C. krusei & 62.5 & 250 & Biofilm formation and & [33] \\
\hline & & C. orthopsilosis & 250 & 2000 & mature biofilm; XTT & [33] \\
\hline & & C. parapsilosis & 500 & 1000 & & \\
\hline & & C. tropicalis & 1000 & 2000 & & \\
\hline Mentha $\times$ piperita $\mathrm{L}$. & $\begin{array}{l}\text { EO: menthol (32.93\%), menthone } \\
(24.41 \%), 1,8 \text {-cineole }(7.89 \%)\end{array}$ & C. albicans & $1-10$ & 10 & Biofilm formation; MTT & {$[44]$} \\
\hline & EO: no composition & C. albicans & 11,600 & 800 & Biofilm formation; XTT & {$[38]$} \\
\hline $\begin{array}{l}\text { Mikania glomerata Spreng } \\
\text { (Asteraceae) }\end{array}$ & $\begin{array}{c}\text { EO: germacrene D }(38.29 \%), \\
\alpha \text {-caryophyllene }(9.49 \%), \\
\text { bicyclogermacrene }(7.98 \%), \\
\text { caryophyllene oxide }(4.28 \%)\end{array}$ & C. albicans & 250 & 500 & $\begin{array}{l}\text { Biofilm formation; } \\
\text { crystal violet }\end{array}$ & [29] \\
\hline
\end{tabular}


Table 1. Cont.

\begin{tabular}{|c|c|c|c|c|c|c|}
\hline $\begin{array}{l}\text { Name of Plant } \\
\text { (Family) }\end{array}$ & $\begin{array}{l}\text { Main Compounds Presented } \\
\text { in the Reference } \\
\text { (EO: Essential Oil) }\end{array}$ & $\begin{array}{l}\text { Targeted } \\
\text { Species of } \\
\text { Candida }\end{array}$ & $\begin{array}{c}\text { MICs } \\
(\mathrm{mg} / \mathrm{L} ; \mathrm{mL} / \mathrm{L})\end{array}$ & $\begin{array}{l}\text { Inhibition of } \\
\text { Biofilm Formation } \\
\text { by at Least } 50 \% \\
(\mathrm{mg} / \mathrm{L} ; \mathrm{mL} / \mathrm{L})\end{array}$ & $\begin{array}{l}\text { Inhibited Stage of } \\
\text { Biofilm; Method of } \\
\text { Biofilm Detection }\end{array}$ & Ref. \\
\hline \multirow{3}{*}{$\begin{array}{l}\text { Myrtus communis L. } \\
\text { (Myrtaceae) }\end{array}$} & \multirow{3}{*}{$\begin{array}{c}\text { EO: } \alpha \text {-pinene }(39.8 \%), 1,8 \text {-cineole }(24.8 \%) \\
\text { limonene }(10.7 \%) \text {, linalool }(6.4 \%)\end{array}$} & C. albicans & $1250-10,000$ & None or 1250 & \multirow{3}{*}{ No data; no data } & \multirow{3}{*}{ [45] } \\
\hline & & C. parapsilosis & $\begin{array}{l}1250 \text { to } \\
>16,000\end{array}$ & 1250 & & \\
\hline & & C. tropicalis & $1250-16,000$ & 1250 & & \\
\hline \multirow{3}{*}{$\begin{array}{l}\text { Ononis spinosa } \mathrm{L} \text {. } \\
\quad \text { (Fabaceae) }\end{array}$} & \multirow{3}{*}{$\begin{array}{l}\text { Extract: kaempherol-O-dihexoside, } \\
\text { kaempherol-O-hexoside-pentoside, } \\
\text { kaempherol-O-hexoside, } \\
\text { quercetin-O-hexoside-pentoside, } \\
\text { acetylquercetin-O-hexoside }\end{array}$} & C. albicans & 620 & 10,000 & \multirow{3}{*}{$\begin{array}{l}\text { Mature biofilm; } \\
\text { luminescence }\end{array}$} & \multirow{3}{*}{ [46] } \\
\hline & & C. krusei & 620 & 5000 & & \\
\hline & & C. tropicalis & 310 & 10,000 & & \\
\hline $\begin{array}{l}\text { Pelargonium graveolens L'Hér. } \\
\text { (Geraniaceae) }\end{array}$ & $\begin{array}{l}\text { EO: geraniol }(42.3 \%) \text {, linalool }(20.1 \%), \\
\text { citronellol }(11.1 \%) \text {, menthone }(8.0 \%)\end{array}$ & C. albicans & 125 & $4000-8000$ & Mature biofilm; XTT & [47] \\
\hline $\begin{array}{l}\text { Piper claussenianum (Miq.) C. } \\
\text { DC. } \\
\text { (Piperaceae) }\end{array}$ & EO: nerolidols & C. albicans & $4100-9600$ & $2400-12,600$ & Mature biofilm; MTT & [48] \\
\hline $\begin{array}{l}\text { Portulaca oleracea L. } \\
\text { (Portulacaceae) }\end{array}$ & Extract: no composition & C. albicans & 10 & 12.5 & Mature biofilm; MTT & [25] \\
\hline $\begin{array}{l}\text { Punica granatum L. } \\
\text { (Lythraceae) }\end{array}$ & Extract: ellagic acid & C. albicans & 1000 & $100-750$ & $\begin{array}{l}\text { Biofilm formation and } \\
\text { mature biofilm; crystal } \\
\text { violet }\end{array}$ & [49] \\
\hline $\begin{array}{l}\text { Santolina impressa Hoffmanns. } \\
\& \text { Link } \\
\text { (Asteraceae) }\end{array}$ & $\begin{array}{c}\text { EO: } \beta \text {-pinene }(22.5 \%), 1,8 \text {-cineole }(10.0 \%), \\
\text { limonene }(9.1 \%), \text { camphor }(8.1 \%) \\
\beta \text {-phellandrene }(8.0 \%)\end{array}$ & C. albicans & 540 & $70-1050$ & Biofilm formation; XTT & [50] \\
\hline $\begin{array}{l}\text { Satureja hortensis L. } \\
\quad \text { (Lamiaceae) }\end{array}$ & $\begin{array}{c}\text { EO: thymol }(45.9 \%) \text {, } \\
\text { gamma-terpinen }(16.71 \%), \text { carvacrol } \\
(12.81 \%), \text { p-cymene }(9.61 \%)\end{array}$ & C. albicans & $200-400$ & $400-4800$ & $\begin{array}{l}\text { Biofilm adhesion, } \\
\text { formation, and mature } \\
\text { biofilm; MTT }\end{array}$ & [51] \\
\hline \multirow{2}{*}{$\begin{array}{c}\text { Satureja macrosiphon (Coss.) }= \\
\text { Micromeria macrosiphon Coss. } \\
\text { (Lamiaceae) }\end{array}$} & \multirow{2}{*}{$\begin{array}{c}\text { EO: linalool }(28.46 \%), \text { borneol }(16.22 \%), \\
\text { terpinene-4-ol }(14.58 \%), \text { cis-sabinene } \\
\text { hydrate }(12.96 \%)\end{array}$} & C. albicans & $0.06-4$ & $0.06-8$ & \multirow[t]{2}{*}{ Biofilm formation; XTT } & \multirow[t]{2}{*}{ [22] } \\
\hline & & C. dubliniensis & $0.25-4$ & $2-8$ & & \\
\hline \multirow{2}{*}{$\begin{array}{c}\text { Syzygium aromaticum }(\mathrm{L} .) \\
\text { Merr. \& L.M.Perry = Eugenia } \\
\text { caryophyllus (Spreng.) } \\
\text { Bullock \& S.G.Harrison } \\
\text { (Myrtaceae) }\end{array}$} & EO: no composition & C. albicans & $100-200$ & 50 & Biofilm formation; XTT & [37] \\
\hline & EO: no composition & C. albicans & 48,000 & 3300 & Biofilm formation; XTT & [38] \\
\hline \multirow{2}{*}{$\begin{array}{l}\text { Thymus vulgaris L. } \\
\text { (Lamiaceae) }\end{array}$} & \multirow{2}{*}{$\begin{array}{c}\text { EO: thymol }(54.73 \%) \text {, carvacrol }(12.42 \%) \text {, } \\
\text { terpineol }(4.00 \%), \text { nerol acetate }(2.86 \%) \\
\text { fenchol }(0.5 \%)\end{array}$} & C. albicans & $1.56-25$ & 12.5 & \multirow{2}{*}{$\begin{array}{l}\text { Biofilm formation; } \\
\text { absorbance, crystal } \\
\text { violet, and scanning } \\
\text { electron microscopy }\end{array}$} & \multirow{2}{*}{ [26] } \\
\hline & & C. tropicalis & $25-50$ & 12.5 & & \\
\hline \multirow{2}{*}{$\begin{array}{l}\text { Warburgia ugandensis Sprague } \\
\text { (Canellaceae) }\end{array}$} & \multirow{2}{*}{$\begin{array}{l}\text { Extract: ugandenial A, warburganal, } \\
\text { polygodial, alpha-linolenic acid ALA }\end{array}$} & C. albicans & Lack of data & 1000 & \multirow{2}{*}{$\begin{array}{l}\text { Biofilm formation and } \\
\text { mature biofilm; XTT } \\
\text { and confocal laser } \\
\text { microscopy }\end{array}$} & \multirow[t]{2}{*}[52]{} \\
\hline & & C. glabrata & Lack of data & 1000 & & \\
\hline $\begin{array}{l}\text { Ziziphora tenuior L. } \\
\text { (Lamiaceae) }\end{array}$ & $\begin{array}{c}\text { EO: pulegone }(46.8 \%), \\
\text { p-menth-3-en-8-ol }(12.5 \%), \\
\text { isomenthone }(6.6 \%) \\
\text { 8-hydroxymenthone }(6.2 \%), \\
\text { isomenthol }(4.7 \%)\end{array}$ & C. albicans & 1.25 & 2.5 & Mature biofilm; XTT & [23] \\
\hline $\begin{array}{l}\text { Zuccagnia punctata } \mathrm{L} . \\
\text { (Fabaceae) }\end{array}$ & Extract: no composition & C. albicans & 400 & 100 & $\begin{array}{l}\text { Biofilm formation and } \\
\text { mature biofilm; XTT } \\
\text { and crystal violet }\end{array}$ & [53] \\
\hline
\end{tabular}

Legend: MIC—minimal inhibitory concentration; XTT—reduction assay of 2,3-bis(2-methoxy-4-nitro-5-sulfophenyl)-5-[carbonyl(phenylamino)]2H-tetrazolium hydroxide; MTT—reduction assay of 3-(4,5-dimethylthiazol-2-yl)-2,5-diphenyltetrazolium bromide [54,55].

Antibiofilm activity may vary between plants in the same family. For example, in the Lamiaceae family, essential oil from Lavandula dentata acted against $C$. albicans biofilm at concentrations of $0.045-0.07 \mu \mathrm{L} / \mathrm{mL}$ [21], while essential oil from Satureja hortensis acted against the same biofilm at concentrations of $400-4800 \mathrm{mg} / \mathrm{L}$ [51]. There may also be large differences within the same species, due to various reasons. This may be influenced by, for example, different research methodologies, the use of different strains of fungi, and different chemical compositions depending on the plant variety, country, and season of harvest. A notable example of such a difference is observed with Mentha $\times$ piperita. In studies by Benzaid et al. [44], essential oil of M. piperita acted against Candida biofilm at a concentration of $10 \mu \mathrm{L} / \mathrm{mL}$. However, the work of Agarwal et al. [38] showed that the same essential oil was active at $800 \mu \mathrm{L} / \mathrm{mL}$.

Changes in the content of active substances were described by Gonçalves et al. [56]. They showed that in essential oil from Mentha cervina collected in August, the amount of 
isomenthone was $8.7 \%$ and pulegone was $75.1 \%$. However, in essential oil collected in February, the ratio of the two compounds reversed and amounted to $77.0 \%$ for isomenthone and $12.9 \%$ for pulegone. The method of obtaining the compounds likewise had an influence on their content in the final essential oil. In a study by Ćavar et al. [57], the composition of essential oils of Calamintha glandulosa differed depending on the extraction method. The level of menthone was 3.3\% using aqueous reflux extraction, $4.7 \%$ using hydrodistillation, and $8.3 \%$ using steam distillation, while the concentration of shisofuran was only $0.1 \%$ using hydrodistillation and steam distillation, while aqueous reflux yielded $9.7 \%$.

\subsection{Plant Compounds That Display Activity against Candida Biofilm}

It has been shown that 69 compounds obtained from plants demonstrate activity against Candida biofilms (Table 2). Among these, the most common are monotherpenes (20), followed by sesquiterpene lactones (7) and sesquiterpenes (6). Another big group is also phenolic compounds, including phenols (6), phenolic acids (5), phenolic aldehydes (2), polyphenols (2), and phenolic alcohol (1).

In terms of activity, large differences were found, depending on the authors cited. Eugenol and thymol serve as good examples. Both compounds exhibited excellent activity in some studies (from $12.5 \mathrm{mg} / \mathrm{L}$ for eugenol [58] and $1.56 \mathrm{mg} / \mathrm{L}$ for thymol [26]), and in other studies, the activity was very poor (up to 80,000 for both [59]). These differences may be related, for example, to a different purity of the compound, a different fungal suspension density, or even to the use of other Candida strains with different sensitivities to chemical substances. A number of other factors, such as the type of culture medium, $\mathrm{pH}$ of the medium, incubation time, and temperature may likewise influence the antimicrobial activity [20].

According to the European Committee on Antimicrobial Susceptibility Testing (EUCAST), the antifungal clinical breakpoints are between $0.001 \mathrm{mg} / \mathrm{L}$ and $16 \mathrm{mg} / \mathrm{L}$ [60]. Using EUCAST guidelines in this review, the most active compounds that inhibit $(>50 \%)$ Candida biofilm formation are lichochalcone A (from $0.2 \mathrm{mg} / \mathrm{L}$ ) [61], thymol (from $3.12 \mathrm{mg} / \mathrm{L}$ ) [26], dioscin (from $3.9 \mathrm{mg} / \mathrm{L}$ ) [31], baicalein (from $4 \mathrm{mg} / \mathrm{L}$ ) [62], warburganal (4.5 mg/L) [52], pterostilbene, waltheriones and riccardin D (both from $8 \mathrm{mg} / \mathrm{L}$ ) [63-65], polygodial (10.8 mg/L) [52], cannabidiol and eugenol (both from $12.5 \mathrm{mg} / \mathrm{L}$ ) [58,66], and ivalin $(15.4 \mathrm{mg} / \mathrm{L})$ [67]. It is interesting that monotherpenes, which represent the highest percentage of substances listed in Table 2, are not the most active compounds. The two larger groups with the best activity are phenolic compounds (thymol, pterostilbene, and eugenol), and sesquiterpene derivatives (warburganal, polygodial, and ivalin). Single compounds with the highest observed activity belong to chalconoids (lichochalcone A), steroidal saponins (dioscin), flavonoids (baicalein), alkaloids (waltheriones), macrocyclic bisbibenzyls (riccardin D), and cannabinoids (cannabidiol). Most of the compounds presented in Table 2 acted on biofilm formation and/or mature biofilm.

Table 2. Antifungal and antibiofilm activity of plant compounds.

\begin{tabular}{|c|c|c|c|c|c|c|}
\hline Active Compound & $\begin{array}{l}\text { Example of Plant } \\
\text { Origin }\end{array}$ & Targeted Fungus & $\begin{array}{c}\mathrm{MICs} \\
(\mathrm{mg} / \mathrm{L}, \mathrm{mL} / \mathrm{L})\end{array}$ & $\begin{array}{c}\text { Inhibition of Biofilm } \\
\text { Formation by at Least } 50 \% \\
(\mathrm{mg} / \mathrm{L}, \mathrm{mL} / \mathrm{L})\end{array}$ & $\begin{array}{l}\text { Inhibited Stage of Biofilm; } \\
\text { Method of Biofilm Detection }\end{array}$ & Ref. \\
\hline \multirow{5}{*}{$\begin{array}{l}\text { Antidesmone } \\
\text { (alkaloid) }\end{array}$} & \multirow{5}{*}{$\begin{array}{l}\text { Waltheria indica, } \\
\text { W. brachypetala }\end{array}$} & C. albicans & 32 & 16 & \multirow{5}{*}{ Mature biofilm; XTT } & \multirow{5}{*}{ [63] } \\
\hline & & C. glabrata & $>32$ & 16 & & \\
\hline & & C. krusei & 16 & 16 & & \\
\hline & & C. parapsilosis & 4 & 16 & & \\
\hline & & C. tropicalis & $>32$ & 16 & & \\
\hline $\begin{array}{c}\text { Anisaldehyde } \\
\text { (phenolic aldehyde) }\end{array}$ & $\begin{array}{l}\text { Pimpinella anisum, } \\
\text { Foeniculum vulgare }\end{array}$ & C. albicans & 500 & 500 & $\begin{array}{l}\text { Mature biofilm; XTT, crystal violet, } \\
\text { and inverted light microscopy }\end{array}$ & [68] \\
\hline $\begin{array}{c}\text { Anisic acid } \\
\text { (phenolic acid) }\end{array}$ & Pimpinella anisum & C. albicans & 4000 & 4000 & $\begin{array}{l}\text { Mature biofilm; XTT, crystal violet, } \\
\text { and inverted light microscopy }\end{array}$ & [68] \\
\hline $\begin{array}{c}\text { Anisyl alcohol } \\
\text { (phenolic alcohol) }\end{array}$ & Pimpinella anisum & C. albicans & 31 & 500 & $\begin{array}{l}\text { Mature biofilm; XTT, crystal violet, } \\
\text { and inverted light microscopy }\end{array}$ & [68] \\
\hline $\begin{array}{l}\text { Baicalein } \\
\text { (flavonoid) }\end{array}$ & $\begin{array}{l}\text { Scutellaria baicalensis, } \\
\text { S. lateriflora }\end{array}$ & C. albicans & No data & $4-32$ & Biofilm formation; XTT & [62] \\
\hline
\end{tabular}


Table 2. Cont.

\begin{tabular}{|c|c|c|c|c|c|c|}
\hline Active Compound & $\begin{array}{l}\text { Example of Plant } \\
\text { Origin }\end{array}$ & Targeted Fungus & $\begin{array}{c}\mathrm{MICs} \\
(\mathrm{mg} / \mathrm{L}, \mathrm{mL} / \mathrm{L})\end{array}$ & $\begin{array}{c}\text { Inhibition of Biofilm } \\
\text { Formation by at Least } 50 \% \\
(\mathrm{mg} / \mathrm{L}, \mathrm{mL} / \mathrm{L})\end{array}$ & $\begin{array}{l}\text { Inhibited Stage of Biofilm; } \\
\text { Method of Biofilm Detection }\end{array}$ & Ref. \\
\hline \multirow{2}{*}{$\begin{array}{c}\text { Camphene } \\
\text { (monotherpene) }\end{array}$} & \multirow{2}{*}{$\begin{array}{l}\text { Croton eluteria, } \\
\text { Cinnamomum verum }\end{array}$} & C. albicans & No data & 500 & $\begin{array}{l}\text { Biofilm formation; confocal laser } \\
\text { microscopy }\end{array}$ & [36] \\
\hline & & C. albicans & 1000 & 2000 & $\begin{array}{l}\text { Mature biofilm; XTT, crystal violet, } \\
\text { and inverted light microscopy }\end{array}$ & [69] \\
\hline \multirow{5}{*}{$\begin{array}{l}\text { Camphor } \\
\text { (bicyclic } \\
\text { monotherpene) }\end{array}$} & \multirow{5}{*}{$\begin{array}{l}\text { Cinnamomum camphora, } \\
\text { Artemisia annua }\end{array}$} & C. albicans & $125-250$ & Not or $62.5-250$ & \multirow{5}{*}{$\begin{array}{l}\text { Biofilm formation; crystal violet } \\
\text { and absorbance }\end{array}$} & \multirow{5}{*}[70]{} \\
\hline & & C. glabrata & 175 & Not & & \\
\hline & & C. krusei & 350 & Not & & \\
\hline & & C. parapsilosis & 125 & Not & & \\
\hline & & C. tropicalis & 175 & 175 & & \\
\hline $\begin{array}{l}\text { Cannabidiol } \\
\text { (cannabinoid) }\end{array}$ & Cannabis sativa & C. albicans & No data & $12.5-100$ & $\begin{array}{l}\text { Biofilm formation; confocal } \\
\text { microscopy }\end{array}$ & [66] \\
\hline \multirow{5}{*}{$\begin{array}{l}\text { Carvacrol } \\
\text { (phenol) }\end{array}$} & \multirow{5}{*}{$\begin{array}{l}\text { Thymus serpyllum, } \\
\text { Carum carvi, } \\
\text { Origanum vulgare }\end{array}$} & \multirow{3}{*}{ C. albicans } & 250 & 500 & $\begin{array}{l}\text { Mature biofilm; XTT, crystal violet, } \\
\text { and inverted light microscopy }\end{array}$ & [69] \\
\hline & & & $100-20,000$ & $300-1250$ & Mature biofilm; XTT & [71] \\
\hline & & & 1000 & $750-1500$ & Biofilm formation; MTT & [72] \\
\hline & & C. glabrata & $100-20,000$ & $300-1250$ & \multirow{2}{*}{ Mature biofilm; XTT } & \multirow{2}{*}{ [71] } \\
\hline & & C. parapsilosis & $100-20,000$ & $300-1250$ & & \\
\hline $\begin{array}{l}\text { Carvene/Limonene } \\
\text { (monotherpene) }\end{array}$ & $\begin{array}{l}\text { Citrus } \times \text { aurantium, } \\
\text { Citrus limon }\end{array}$ & C. albicans & 1000 & 4000 & $\begin{array}{l}\text { Mature biofilm; XTT, crystal violet, } \\
\text { and inverted light microscopy }\end{array}$ & [69] \\
\hline $\begin{array}{l}\text { Carvone/Carvol } \\
\text { (monotherpene) }\end{array}$ & $\begin{array}{l}\text { Carum carvi, } \\
\text { Mentha spicata }\end{array}$ & C. albicans & $>4000$ & 250 & $\begin{array}{l}\text { Mature biofilm; XTT, crystal violet, } \\
\text { and inverted light microscopy }\end{array}$ & [69] \\
\hline $\begin{array}{l}\beta \text {-Caryophyllene } \\
\text { (sesquiterpene) }\end{array}$ & $\begin{array}{l}\text { Helichrysum italicum, } \\
\text { Caryophyllusaromaticus }\end{array}$ & C. albicans & No data & $100-500$ & $\begin{array}{l}\text { Biofilm formation; confocal laser } \\
\text { microscopy }\end{array}$ & [36] \\
\hline $\begin{array}{c}\text { 1,4-Cineole } \\
\text { (monotherpene) }\end{array}$ & $\begin{array}{l}\text { Rosmarinus officinalis, } \\
\text { Thymus vulgaris }\end{array}$ & C. albicans & $>4000$ & 4000 & $\begin{array}{l}\text { Mature biofilm; XTT, crystal violet, } \\
\text { and inverted light microscopy }\end{array}$ & [69] \\
\hline \multirow{7}{*}{$\begin{array}{l}1,8- \\
\text { Cineole/Eucalyptol } \\
\text { (monotherpene) }\end{array}$} & \multirow{7}{*}{$\begin{array}{l}\text { Eucalyptus globulus, } \\
\text { Salvia officinalis, } \\
\text { Pinus sylvestris }\end{array}$} & \multirow{3}{*}{ C. albicans } & 4000 & 4000 & $\begin{array}{l}\text { Mature biofilm; XTT, crystal violet, } \\
\text { and inverted light microscopy }\end{array}$ & [69] \\
\hline & & & 8 & 4 & Mature biofilm; luminescence & [40] \\
\hline & & & $3000-23,000$ & Not or $3000-23,000$ & \multirow{5}{*}{$\begin{array}{l}\text { Biofilm formation; crystal violet } \\
\text { and absorbance }\end{array}$} & \multirow{5}{*}[70]{} \\
\hline & & C. glabrata & 2000 & Not & & \\
\hline & & C. krusei & 4000 & $2000-4000$ & & \\
\hline & & C. parapsilosis & 2000 & $1000-2000$ & & \\
\hline & & C. tropicalis & 4000 & $2000-4000$ & & \\
\hline \multirow{2}{*}{$\begin{array}{l}\text { Cinnamaldehyde } \\
\text { (aldehyde) }\end{array}$} & \multirow{2}{*}{$\begin{array}{l}\text { Cinnamomum sp., } \\
\text { Apium graveolens }\end{array}$} & \multirow{2}{*}{ C. albicans } & 62 & 125 & $\begin{array}{l}\text { Mature biofilm; XTT, crystal violet, } \\
\text { and inverted light microscopy }\end{array}$ & [68] \\
\hline & & & $50-400$ & $25-200$ & Mature biofilm; XTT & [58] \\
\hline $\begin{array}{l}\text { Cinnamic acid } \\
\text { (phenolic acid) }\end{array}$ & Cinnamomum sp. & C. albicans & 2000 & 4000 & $\begin{array}{l}\text { Mature biofilm; XTT, crystal violet, } \\
\text { and inverted light microscopy }\end{array}$ & [68] \\
\hline $\begin{array}{c}\text { Citral } \\
\text { (monotherpene) }\end{array}$ & $\begin{array}{l}\text { Melissa officinalis, } \\
\text { Backhousia citriodora }\end{array}$ & C. albicans & 500 & 1000 & $\begin{array}{l}\text { Mature biofilm; XTT, crystal violet, } \\
\text { and inverted light microscopy }\end{array}$ & [69] \\
\hline $\begin{array}{c}\text { Citronellal } \\
\text { (monotherpene) }\end{array}$ & $\begin{array}{l}\text { Cymbopogon citratus } \\
\text { Melissa officinalis }\end{array}$ & C. albicans & 500 & 1000 & $\begin{array}{l}\text { Mature biofilm; XTT, crystal violet, } \\
\text { and inverted light microscopy }\end{array}$ & [69] \\
\hline $\begin{array}{c}\beta \text {-Citronellol } \\
\text { (monotherpene) }\end{array}$ & $\begin{array}{l}\text { Melissa officinalis, } \\
\text { Pelargonium roseum }\end{array}$ & C. albicans & 500 & 1000 & $\begin{array}{l}\text { Mature biofilm; XTT, crystal violet, } \\
\text { and inverted light microscopy }\end{array}$ & [69] \\
\hline $\begin{array}{l}\text { Cuminaldehyde } \\
\text { (monotherpene) }\end{array}$ & $\begin{array}{l}\text { Carum carvi } \\
\text { Cinnamomum verum }\end{array}$ & C. albicans & 1000 to $>4000$ & $6000-7000$ & Biofilm formation; MTT & [72] \\
\hline $\begin{array}{c}\text { p-Cymene } \\
\text { (monotherpene) }\end{array}$ & $\begin{array}{l}\text { Thymus vulgaris, } \\
\text { Eucalyptus sp. }\end{array}$ & C. albicans & 2000 & 4000 & $\begin{array}{l}\text { Mature biofilm; XTT, crystal violet, } \\
\text { and inverted light microscopy }\end{array}$ & [69] \\
\hline \multirow{5}{*}{$\begin{array}{l}\text { 8-Deoxoantidesmone } \\
\text { (alkaloid) }\end{array}$} & \multirow{5}{*}{ Waltheria indica } & C. albicans & 16 & 32 & \multirow{5}{*}{ Mature biofilm; XTT } & \multirow{5}{*}{ [63] } \\
\hline & & C. glabrata & $>32$ & 32 & & \\
\hline & & C. krusei & 32 & 32 & & \\
\hline & & C. parapsilosis & 32 & 32 & & \\
\hline & & C. tropicalis & $>32$ & 32 & & \\
\hline $\begin{array}{c}2^{\prime}, 4^{\prime} \text {-Dihydroxy-3'- } \\
\text { methoxychalcone } \\
\text { (chalcone) }\end{array}$ & $\begin{array}{l}\text { Zuccagnia punctata, } \\
\text { Oxytropis falcata }\end{array}$ & C. albicans & 100 & 25 & $\begin{array}{l}\text { Biofilm formation and mature } \\
\text { biofilm; XTT and crystal violet }\end{array}$ & [53] \\
\hline $\begin{array}{c}\text { Dioscin } \\
\text { (steroidal saponin) }\end{array}$ & $\begin{array}{l}\text { Dioscorea sp., } \\
\text { Chamaecostus }\end{array}$ & C. albicans & $3.9-15.62$ & $3.9-31.25$ & $\begin{array}{l}\text { Biofilm formation and mature } \\
\text { biofilm; MTT }\end{array}$ & [31] \\
\hline $\begin{array}{l}\text { Ellagic acid } \\
\text { (polyphenol) }\end{array}$ & Punica granatum $\mathrm{L}$. & C. albicans & $75-100$ & $25-40$ & $\begin{array}{c}\text { Biofilm formation and mature } \\
\text { biofilm; crystal violet }\end{array}$ & [49] \\
\hline $\begin{array}{c}\text { Emodin } \\
\text { (anthraquinone) }\end{array}$ & $\begin{array}{l}\text { Rheum palmatum, } \\
\text { Frangula alnus }\end{array}$ & C. albicans & $12.5-50$ & Not or $100-400$ & Biofilm adhesion; MTT & [73] \\
\hline
\end{tabular}


Table 2. Cont.

\begin{tabular}{|c|c|c|c|c|c|c|}
\hline Active Compound & Example of Plant Origin & Targeted Fungus & $\begin{array}{c}\text { MICs } \\
(\mathrm{mg} / \mathrm{L}, \mathrm{mL} / \mathrm{L})\end{array}$ & $\begin{array}{c}\text { Inhibition of Biofilm } \\
\text { Formation by at Least } 50 \% \\
(\mathrm{mg} / \mathrm{L}, \mathrm{mL} / \mathrm{L})\end{array}$ & $\begin{array}{l}\text { Inhibited Stage of Biofilm; Method } \\
\text { of Biofilm Detection }\end{array}$ & Ref. \\
\hline $\begin{array}{c}4 \alpha, 5 \alpha \text {-Epoxy- } \\
10 \alpha, 14 \mathrm{H}-1 \text {-epi- } \\
\text { inuviscolide } \\
\text { (sesquiterpene lactone) }\end{array}$ & Carpesium macrocephalum & C. albicans & $>128$ & 38 & $\begin{array}{l}\text { Biofilm formation and mature } \\
\text { biofilm; XTT }\end{array}$ & [67] \\
\hline \multirow{4}{*}{$\begin{array}{l}\text { Eugenol } \\
\text { (phenol) }\end{array}$} & \multirow{4}{*}{$\begin{array}{l}\text { Syzygium aromaticum, } \\
\text { Cinnamomum sp. }\end{array}$} & \multirow{4}{*}{ C. albicans } & $50-400$ & $12.5-200$ & Mature biofilm; XTT & [58] \\
\hline & & & 250 & 500 & $\begin{array}{l}\text { Mature biofilm; XTT, crystal violet, } \\
\text { and inverted light microscopy }\end{array}$ & [69] \\
\hline & & & 500 & 500 & $\begin{array}{l}\text { Mature biofilm; XTT, crystal violet, } \\
\text { and inverted light microscopy }\end{array}$ & [68] \\
\hline & & & 1200 & $10,000-80,000$ & Mature biofilm; XTT & [59] \\
\hline \multirow[t]{2}{*}{$\begin{array}{c}\text { Farnesol } \\
\text { (sesquiterpene) }\end{array}$} & \multirow[t]{2}{*}{$\begin{array}{l}\text { Tilia sp., } \\
\text { Cymbopogon sp. }\end{array}$} & \multirow[t]{2}{*}{ C. albicans } & 1000 & 500 & $\begin{array}{l}\text { Mature biofilm; XTT, crystal violet, } \\
\text { and inverted light microscopy }\end{array}$ & [68] \\
\hline & & & 1000 & 500 & $\begin{array}{l}\text { Mature biofilm; XTT, crystal violet, } \\
\text { and inverted light microscopy }\end{array}$ & [69] \\
\hline $\begin{array}{c}\text { Gallic acid } \\
\text { (phenolic acid) }\end{array}$ & $\begin{array}{l}\text { Polygonum sp., } \\
\text { Buchenavia tomentosa }\end{array}$ & C. albicans & 5000 & 2500 & $\begin{array}{l}\text { Biofilm formation and mature } \\
\text { biofilm; culture }\end{array}$ & [30] \\
\hline \multirow{5}{*}{$\begin{array}{c}\text { Geraniol } \\
\text { (monotherpene) }\end{array}$} & \multirow{5}{*}{$\begin{array}{l}\text { Pelargonium graveolens, } \\
\text { Rosa sp. }\end{array}$} & C. albicans & 1000 & 1000 & $\begin{array}{l}\text { Mature biofilm; XTT, crystal violet, } \\
\text { and inverted light microscopy }\end{array}$ & [69] \\
\hline & & C. albicans & $100-20,000$ & $300-1250$ & Mature biofilm; XTT & [71] \\
\hline & & C. albicans & No data & $1000-8000$ & Mature biofilm; XTT & [47] \\
\hline & & C. glabrata & $100-20,000$ & $300-1250$ & \multirow[t]{2}{*}{ Mature biofilm; XTT } & \multirow[t]{2}{*}{ [71] } \\
\hline & & C. parapsilosis & $100-20,000$ & $300-1250$ & & \\
\hline $\begin{array}{l}\text { Guaiacol } \\
\text { (phenol) }\end{array}$ & $\begin{array}{l}\text { Guaiacum officinale, } \\
\text { Apium graveolens }\end{array}$ & C. albicans & 500 & 1000 & $\begin{array}{l}\text { Mature biofilm; XTT, crystal violet, } \\
\text { and inverted light microscopy }\end{array}$ & [68] \\
\hline $\begin{array}{l}\text { Hydroxychavicol } \\
\text { (phenol) }\end{array}$ & Piper betle & C. albicans & $125-500$ & $125-1000$ & $\begin{array}{l}\text { Biofilm formation and mature } \\
\text { biofilm; XTT }\end{array}$ & [74] \\
\hline $\begin{array}{c}\beta \text {-Ionone } \\
\text { (carotenoid) }\end{array}$ & $\begin{array}{l}\text { Lawsonia inermis, } \\
\text { Camellia sinensis }\end{array}$ & C. albicans & 250 & 250 & $\begin{array}{l}\text { Mature biofilm; XTT, crystal violet, } \\
\text { and inverted light microscopy }\end{array}$ & [69] \\
\hline \multirow{2}{*}{$\begin{array}{c}\text { Isomontanolide } \\
\text { (sesquiterpenic } \\
\text { lactone) }\end{array}$} & \multirow{2}{*}{$\begin{array}{l}\text { Laserpitium ochridanum, } \\
\text { L. zernyi }\end{array}$} & C. albicans & 50 & 250 & \multirow{2}{*}{ Mature biofilm; luminescence } & \multirow{2}{*}{ [43] } \\
\hline & & C. krusei & 200 & 250 & & \\
\hline $\begin{array}{c}\text { Isopulegol } \\
\text { (monotherpene) }\end{array}$ & $\begin{array}{l}\text { Mentha rotundifolia, } \\
\text { Melissa officinalis }\end{array}$ & C. albicans & $>4000$ & 250 & $\begin{array}{l}\text { Mature biofilm; XTT, crystal violet, } \\
\text { and inverted light microscopy }\end{array}$ & [69] \\
\hline $\begin{array}{c}\text { Ivalin } \\
\text { (sesquiterpene lactone) }\end{array}$ & $\begin{array}{c}\text { Geigeria aspera, } \\
\text { Carpesium macrocephalum }\end{array}$ & C. albicans & $>128$ & 15.4 & $\begin{array}{l}\text { Biofilm formation and mature } \\
\text { biofilm; XTT }\end{array}$ & [67] \\
\hline \multirow{2}{*}{$\begin{array}{c}\text { Laserpitine } \\
\text { (sesquiterpene lactone) }\end{array}$} & \multirow{2}{*}{$\begin{array}{l}\text { Laserpitium latifolium, } \\
\text { Laserpitiumhalleri }\end{array}$} & C. albicans & 200 & 400 & \multirow[t]{2}{*}{ Mature biofilm; luminescence } & \multirow{2}{*}{ [43] } \\
\hline & & C. krusei & 200 & 400 & & \\
\hline $\begin{array}{l}\text { Lichochalcone A } \\
\text { (chalconoid) }\end{array}$ & Glycyrrhiza sp. & C. albicans & $6.25-12.5$ & $0.2-20$ & Biofilm formation; crystal violet & [61] \\
\hline \multirow{3}{*}{$\begin{array}{c}\text { Linalool } \\
\text { (monotherpene) }\end{array}$} & \multirow{3}{*}{$\begin{array}{l}\text { Lavandula officinalis, } \\
\text { Pelargonium graveolens }\end{array}$} & \multirow{3}{*}{ C. albicans } & No data & $100-500$ & $\begin{array}{l}\text { Biofilm formation; confocal laser } \\
\text { microscopy }\end{array}$ & [36] \\
\hline & & & 2000 & 1000 & $\begin{array}{l}\text { Mature biofilm; XTT, crystal violet, } \\
\text { and inverted light microscopy }\end{array}$ & [69] \\
\hline & & & No data & $1000-8000$ & Mature biofilm; XTT & [47] \\
\hline $\begin{array}{l}\alpha \text {-Longipinene } \\
\text { (sesquiterpene) }\end{array}$ & $\begin{array}{c}\text { Croton eluteria, } \\
\text { Helichrysum italicum }\end{array}$ & C. albicans & No data & $100-500$ & $\begin{array}{l}\text { Biofilm formation; confocal laser } \\
\text { microscopy }\end{array}$ & [36] \\
\hline \multirow[t]{2}{*}{$\begin{array}{c}\text { Menthol } \\
\text { (monotherpene) }\end{array}$} & \multirow[t]{2}{*}{ Mentha spp. } & \multirow[t]{2}{*}{ C. albicans } & $>4000$ & 2000 & $\begin{array}{l}\text { Mature biofilm; XTT, crystal violet, } \\
\text { and inverted light microscopy }\end{array}$ & [69] \\
\hline & & & 2500 & $10,000-80,000$ & Mature biofilm; XTT & [59] \\
\hline \multirow{2}{*}{$\begin{array}{c}\text { Montanolide } \\
\text { (sesquiterpene lactone) }\end{array}$} & Laserpitium ochridanum, & C. albicans & 200 & 400 & Mature biofilm; luminescence & [43] \\
\hline & & C. krusei & 200 & 400 & & \\
\hline $\begin{array}{c}\text { Morin } \\
\text { (flavonoid) }\end{array}$ & $\begin{array}{l}\text { Prunus dulcis } \\
\text { Morus alba }\end{array}$ & C. albicans & 150 & $37.5-600$ & Biofilm formation; crystal violet & [75] \\
\hline $\begin{array}{c}\text { Myrcene } \\
\text { (monotherpene) }\end{array}$ & $\begin{array}{l}\text { Humulus lupulus, } \\
\text { Cannabis sativa }\end{array}$ & C. albicans & 1000 & 2000 & $\begin{array}{l}\text { Mature biofilm; XTT, crystal violet, } \\
\text { and inverted light microscopy }\end{array}$ & [69] \\
\hline $\begin{array}{c}\text { Nerol } \\
\text { (monotherpene) }\end{array}$ & $\begin{array}{l}\text { Citrus } \times \text { aurantium, } \\
\text { Humulus lupulus }\end{array}$ & C. albicans & 2000 & 500 & $\begin{array}{l}\text { Mature biofilm; XTT, crystal violet, } \\
\text { and inverted light microscopy }\end{array}$ & [69] \\
\hline $\begin{array}{c}\text { Nerolidols } \\
\text { (sesquiterpene) }\end{array}$ & $\begin{array}{l}\text { Citrus } \times \text { aurantium, } \\
\text { Piper claussenianum }\end{array}$ & C. albicans & $18,600-62,500$ & $2500-10,000$ & Mature biofilm; MTT & [48] \\
\hline $\begin{array}{c}\alpha \text {-Pinene } \\
\text { (monotherpene) }\end{array}$ & $\begin{array}{l}\text { Pinus sylvestris, } \\
\text { Picea abies }\end{array}$ & C. albicans & 3125 & 3125 & Biofilm formation; XTT & [76] \\
\hline $\begin{array}{c}\beta \text {-Pinene } \\
\text { (monotherpene) }\end{array}$ & $\begin{array}{l}\text { Pinus sylvestris, } \\
\text { Picea abies }\end{array}$ & C. albicans & 2000 & 4000 & $\begin{array}{l}\text { Mature biofilm; XTT, crystal violet, } \\
\text { and inverted light microscopy }\end{array}$ & [69] \\
\hline & & & 187 & 187 & Biofilm formation; XTT & [76] \\
\hline Polygodial & Warburgia ugandensis, & C. albicans & 4.1 & 10.8 & Biofilm formation and mature & [52] \\
\hline (sesquiter & Polygonun & C. glabrata & 94.1 & $50.6-61.9$ & $\begin{array}{l}\text { b1ofilm; } X 11 \text { and confocal laser } \\
\text { microscopy }\end{array}$ & \\
\hline
\end{tabular}


Table 2. Cont.

\begin{tabular}{|c|c|c|c|c|c|c|}
\hline Active Compound & Example of Plant Origin & Targeted Fungus & $\underset{(\mathrm{mg} / \mathrm{L}, \mathrm{mL} / \mathrm{L})}{\mathrm{MICs}}$ & $\begin{array}{c}\text { Inhibition of Biofilm } \\
\text { Formation by at Least } 50 \% \\
(\mathrm{mg} / \mathrm{L}, \mathrm{mL} / \mathrm{L})\end{array}$ & $\begin{array}{l}\text { Inhibited Stage of Biofilm; Method } \\
\text { of Biofilm Detection }\end{array}$ & Ref. \\
\hline $\begin{array}{l}\text { Pterostilbene } \\
\text { (polyphenol) }\end{array}$ & $\begin{array}{l}\text { Pterocarpus marsupium, } \\
\text { Pterocarpus santalinus, } \\
\text { Vitis vinifera }\end{array}$ & C. albicans & No data & $8-32$ & $\begin{array}{l}\text { Biofilm formation and mature } \\
\text { biofilm; XTT }\end{array}$ & [65] \\
\hline $\begin{array}{l}\text { Riccardin D } \\
\text { (macrocyclic } \\
\text { bisbibenzyl) }\end{array}$ & Dumortiera hirsuta & C. albicans & 16 & $8-64$ & Mature biofilm; XTT & [64] \\
\hline $\begin{array}{c}\text { Salicylaldehyde } \\
\text { (phenolic aldehyde) }\end{array}$ & $\begin{array}{l}\text { Filipendula ulmaria, } \\
\text { Fagopyrum esculentum }\end{array}$ & C. albicans & 31 & 125 & $\begin{array}{l}\text { Mature biofilm; XTT, crystal violet, } \\
\text { and inverted light microscopy }\end{array}$ & [68] \\
\hline $\begin{array}{l}\text { Salicylic acid } \\
\text { (phenolic acid) }\end{array}$ & $\begin{array}{c}\text { Salix sp., } \\
\text { Filipendula ulmaria }\end{array}$ & C. albicans & 4000 & 2000 & $\begin{array}{l}\text { Mature biofilm; XTT, crystal violet, } \\
\text { and inverted light microscopy }\end{array}$ & [68] \\
\hline $\begin{array}{l}\text { Scopoletin } \\
\text { (cumarin) }\end{array}$ & $\begin{array}{l}\text { Mitracarpus frigidus, } \\
\text { Scopolia carniola }\end{array}$ & C. tropicalis & 50 & 50 & $\begin{array}{l}\text { Biofilm adhesion, formation, and } \\
\text { mature biofilm; absorbance and } \\
\text { digital scanning }\end{array}$ & [77] \\
\hline $\begin{array}{c}\text { 6-Shogaol } \\
\text { (phenylalkane) }\end{array}$ & Zingiber officinale & C. auris & $32-64$ & $16-64$ & Mature biofilm; crystal violet & [78] \\
\hline \multirow{2}{*}{$\begin{array}{c}\text { Tarolide } \\
\text { (sesquiterpene lactone) }\end{array}$} & \multirow{2}{*}{$\begin{array}{l}\text { Laserpitium ochridanum, } \\
\text { L. zernyi }\end{array}$} & C. albicans & 400 & 1000 & \multirow{2}{*}{ Mature biofilm; luminescence } & \multirow{2}{*}{ [43] } \\
\hline & & C. krusei & 400 & 1000 & & \\
\hline $\begin{array}{c}\text { Telekin } \\
\text { (sesquiterpene lactone) }\end{array}$ & $\begin{array}{l}\text { Carpesium macrocephalum, } \\
\text { Telekia speciose }\end{array}$ & C. albicans & $>128$ & 36 & $\begin{array}{l}\text { Biofilm formation and mature } \\
\text { biofilm; XTT }\end{array}$ & [67] \\
\hline $\begin{array}{l}\text { Terpinolene } \\
\text { (terpene) }\end{array}$ & $\begin{array}{l}\text { Cannabis sativa, } \\
\text { Citrus limon }\end{array}$ & C. albicans & 2000 & 4000 & $\begin{array}{l}\text { Mature biofilm; XTT, crystal violet, } \\
\text { and inverted light microscopy }\end{array}$ & [69] \\
\hline $\begin{array}{c}5,7,3^{\prime}, 4^{\prime}- \\
\text { Tetramethoxyflavone } \\
\text { (flavonoid) }\end{array}$ & $\begin{array}{l}\text { Psiadia punctulate, } \\
\text { Kaempferia parviflora }\end{array}$ & C. albicans & 100 & 40 & Biofilm formation; crystal violet & [79] \\
\hline $\begin{array}{c}\alpha \text {-Thujone } \\
\text { (monotherpene) }\end{array}$ & $\begin{array}{l}\text { Artemisia absinthium, } \\
\text { Tanacetum vulgare }\end{array}$ & C. albicans & $>4000$ & 500 & $\begin{array}{l}\text { Mature biofilm; XTT, crystal violet, } \\
\text { and inverted light microscopy }\end{array}$ & [69] \\
\hline \multirow{9}{*}{$\begin{array}{l}\text { Thymol } \\
\text { (phenol) }\end{array}$} & \multirow{9}{*}{$\begin{array}{l}\text { Thymus vulgaris, } \\
\text { Trachyspermum copticum }\end{array}$} & \multirow{6}{*}{ C. albicans } & 250 & 250 & $\begin{array}{l}\text { Mature biofilm; XTT, crystal violet, } \\
\text { and inverted light microscopy }\end{array}$ & [69] \\
\hline & & & $1.56-50$ & 3.12 & $\begin{array}{c}\text { Biofilm formation; absorbance, } \\
\text { crystal violet, and scanning electron } \\
\text { microscopy }\end{array}$ & [26] \\
\hline & & & $32-128$ & 128 & $\begin{array}{l}\text { Biofilm adhesion and mature } \\
\text { biofilm; XTT }\end{array}$ & [80] \\
\hline & & & $100-20,000$ & $300-1250$ & Mature biofilm; XTT & [71] \\
\hline & & & 125 & $125-250$ & $\begin{array}{c}\text { Biofilm formation and mature } \\
\text { biofilm; XTT }\end{array}$ & [81] \\
\hline & & & 1200 & $5000-80,000$ & Mature biofilm; XTT & [59] \\
\hline & & C. tropicalis & $1.56-50$ & 12.5 & $\begin{array}{l}\text { Biofilm formation; absorbance, } \\
\text { crystal violet, and scanning electron } \\
\text { microscopy }\end{array}$ & [26] \\
\hline & & C. glabrata & $100-20,000$ & $300-1250$ & \multirow{2}{*}{ Mature biofilm; XTT } & \multirow{2}{*}{ [71] } \\
\hline & & C. parapsilosis & $100-20,000$ & $300-1250$ & & \\
\hline $\begin{array}{l}\text { Tn-AFP1 } \\
\text { (protein) }\end{array}$ & Trapa natans & C. tropicalis & 32 & 16 & Mature biofilm; XTT & [82] \\
\hline $\begin{array}{c}\text { 5,6,8-Trihydroxy-7,4' } \\
\text { dimethoxy flavone } \\
\text { (flavonoid) }\end{array}$ & $\begin{array}{c}\text { Thymus membranaceus } \\
\text { subsp. membranaceus, } \\
\text { Dodonaea viscosa var. } \\
\text { angustifolia }\end{array}$ & C. albicans & 390 & 390 & $\begin{array}{l}\text { Biofilm formation and mature } \\
\text { biofilm; MTT }\end{array}$ & [83] \\
\hline \multirow{5}{*}{$\begin{array}{l}\text { 5(R)-Vanessine } \\
\text { (alkaloid) }\end{array}$} & \multirow{5}{*}{ Waltheria indica } & C. albicans & 32 & 16 & \multirow{5}{*}{ Mature biofilm; XTT } & \multirow{5}{*}{ [63] } \\
\hline & & C. glabrata & $>32$ & 16 & & \\
\hline & & C. krusei & 32 & 16 & & \\
\hline & & C. parapsilosis & $>32$ & 16 & & \\
\hline & & C. tropicalis & $>32$ & 16 & & \\
\hline $\begin{array}{l}\text { Vanillic acid } \\
\text { (phenolic acid) }\end{array}$ & $\begin{array}{l}\text { Angelica sinensis, } \\
\text { Solanum tuberosum }\end{array}$ & C. albicans & $>4000$ & 4000 & $\begin{array}{l}\text { Mature biofilm; XTT, crystal violet, } \\
\text { and inverted light microscopy }\end{array}$ & [68] \\
\hline $\begin{array}{l}\text { Vanillin } \\
\text { (phenol) }\end{array}$ & Vanilla planifolia & C. albicans & 1000 & 500 & $\begin{array}{l}\text { Mature biofilm; XTT, crystal violet, } \\
\text { and inverted light microscopy }\end{array}$ & [68] \\
\hline \multirow{5}{*}{$\begin{array}{l}\text { Waltheriones } \\
\text { (alkaloid) }\end{array}$} & \multirow{5}{*}{$\begin{array}{l}\text { Waltheria indica, } \\
\text { W. viscosissima }\end{array}$} & C. albicans & $4-32$ & $8-32$ & \multirow{5}{*}{ Mature biofilm; XTT } & \multirow{5}{*}{ [63] } \\
\hline & & C. glabrata & 32 or $>32$ & $8-32$ & & \\
\hline & & C. krusei & $16-32$ or $>32$ & $8-32$ & & \\
\hline & & C. parapsilosis & $2-32$ or $>32$ & $8-32$ & & \\
\hline & & C. tropicalis & 32 or $>32$ & $8-32$ & & \\
\hline \multirow{2}{*}{$\begin{array}{l}\text { Warburganal } \\
\text { (sesquiterpene) }\end{array}$} & \multirow{2}{*}{ Warburgia sp. } & C. albicans & 4 & 4.5 & \multirow{2}{*}{$\begin{array}{l}\text { Biofilm formation and mature } \\
\text { biofilm; XTT and confocal laser } \\
\text { microscopy }\end{array}$} & \multirow{2}{*}{ [52] } \\
\hline & & C. glabrata & $72-72.6$ & $49.1-55.9$ & & \\
\hline
\end{tabular}

Legend: MIC-minimal inhibitory concentration; XTT-reduction assay of 2,3-bis(2-methoxy-4-nitro-5-sulfophenyl)-5[carbonyl(phenylamino)]-2H-tetrazolium hydroxide; MTT-reduction assay of 3-(4,5-dimethylthiazol-2-yl)-2,5-diphenyltetrazolium bromide [54,55]. 


\section{Conclusions}

Plant preparations (essential oils and extracts) and pure compounds exhibit antibiofilm activity against Candida species. Some of them are characterized by high activity in concentrations below $16 \mathrm{mg} / \mathrm{L}$. Given this activity at relatively low concentrations, some may prove to be promising alternatives to antifungal drugs, especially in the cases of resistant or multiresistant strains of Candida. Moreover, the simple chemical structures involved and relative ease of extraction from natural sources warrant further research into the development of new, promising, and much-needed plant-based antifungals.

Author Contributions: Conceptualization, T.M.K. and M.O.; methodology, T.M.K.; analysis of results, T.M.K. and M.O.; writing—original draft preparation, T.M.K., M.O., A.S.-M., H.W., and A.A.; writing-review and editing, T.M.K. and M.O.; supervision, T.M.K.; funding acquisition, T.M.K. and H.W. All authors have read and agreed to the published version of the manuscript.

Funding: This research received no external funding.

Institutional Review Board Statement: Not applicable.

Informed Consent Statement: Not applicable.

Acknowledgments: We are very grateful to Mark Stasiewicz for English language corrections.

Conflicts of Interest: The authors declare no conflict of interest.

\section{References}

1. Brown, G.D.; Denning, D.W.; Gow, N.A.R.; Levitz, S.M.; Netea, M.G.; White, T.C. Hidden Killers: Human Fungal Infections. Sci. Transl. Med. 2012, 4, 165rv13. [CrossRef]

2. Ciurea, C.N.; Kosovski, I.-B.; Mare, A.D.; Toma, F.; Pintea-Simon, I.A.; Man, A. Candida and Candidiasis-Opportunism Versus Pathogenicity: A Review of the Virulence Traits. Microorganisms 2020, 8, 857. [CrossRef] [PubMed]

3. Moran, G.; Coleman, D.; Sullivan, D. An Introduction to the Medically Important Candida Species. In Candida and Candidiasis, 2nd ed.; Wiley: Hoboken, NJ, USA, 2012; pp. 11-25.

4. Buranarom, N.; Komin, O.; Matangkasombut, O. Hyposalivation, Oral Health, and Candida Colonization in Independent Dentate Elders. PLoS ONE 2020, 15, e242832. [CrossRef] [PubMed]

5. Arya, N.R.; Naureen, R.B. Candidiasis. In StatPearls; StatPearls Publishing: Treasure Island, FL, USA, 2021.

6. Millet, N.; Solis, N.V.; Swidergall, M. Mucosal IgA Prevents Commensal Candida Albicans Dysbiosis in the Oral Cavity. Front. Immunol. 2020, 11, 555363. [CrossRef] [PubMed]

7. Sobel, J.D. Vulvovaginal Candidosis. Lancet 2007, 369, 1961-1971. [CrossRef]

8. Vila, T.; Sultan, A.S.; Montelongo-Jauregui, D.; Jabra-Rizk, M.A. Oral Candidiasis: A Disease of Opportunity. J. Fungi $2020,6,15$. [CrossRef] [PubMed]

9. Karpiński, T.; Sopata, M.; Mańkowski, B. The Antimicrobial Effectiveness of Antiseptics as a Challenge in Hard to Heal Wounds. Leczenie Ran 2020, 17, 88-94. [CrossRef]

10. Bhattacharya, S.; Sae-Tia, S.; Fries, B.C. Candidiasis and Mechanisms of Antifungal Resistance. Antibiotics 2020, 9, 312. [CrossRef]

11. Cornely, O.A.; Bassetti, M.; Calandra, T.; Garbino, J.; Kullberg, B.J.; Lortholary, O.; Meersseman, W.; Akova, M.; Arendrup, M.C.; Arikan-Akdagli, S.; et al. ESCMID* Guideline for the Diagnosis and Management of Candida Diseases 2012: Non-Neutropenic Adult Patients. Clin. Microbiol. Infect. 2012, 18 (Suppl. 7), 19-37. [CrossRef]

12. Pappas, P.G.; Kauffman, C.A.; Andes, D.R.; Clancy, C.J.; Marr, K.A.; Ostrosky-Zeichner, L.; Reboli, A.C.; Schuster, M.G.; Vazquez, J.A.; Walsh, T.J.; et al. Clinical Practice Guideline for the Management of Candidiasis: 2016 Update by the Infectious Diseases Society of America. Clin. Infect. Dis. 2016, 62, e1-e50. [CrossRef]

13. Marak, M.B.; Dhanashree, B. Antifungal Susceptibility and Biofilm Production of Candida Spp. Isolated from Clinical Samples. Int. J. Microbiol. 2018, 2018, 7495218. [CrossRef] [PubMed]

14. Rudramurthy, S.M.; Chakrabarti, A.; Paul, R.A.; Sood, P.; Kaur, H.; Capoor, M.R.; Kindo, A.J.; Marak, R.S.K.; Arora, A.; Sardana, R.; et al. Candida auris Candidaemia in Indian ICUs: Analysis of Risk Factors. J. Antimicrob. Chemother. 2017, 72, $1794-1801$. [CrossRef] [PubMed]

15. Mayer, F.L.; Wilson, D.; Hube, B. Candida Albicans Pathogenicity Mechanisms. Virulence 2013, 4, 119-128. [CrossRef] [PubMed]

16. Łaska, G.; Sienkiewicz, A. Antifungal Activity of the Rhizome Extracts of Pulsatilla Vulgaris against Candida Glabrata. Eur. J. Biol. Res. 2019, 9, 93-103.

17. Gebreyohannes, G.; Nyerere, A.; Bii, C.; Sbhatu, D.B. Challenges of Intervention, Treatment, and Antibiotic Resistance of Biofilm-Forming Microorganisms. Heliyon 2019, 5, e02192. [CrossRef]

18. Pereira, R.; Dos Santos Fontenelle, R.O.; de Brito, E.H.S.; de Morais, S.M. Biofilm of Candida Albicans: Formation, Regulation and Resistance. J. Appl. Microbiol. 2020. [CrossRef] [PubMed] 
19. Tumbarello, M.; Fiori, B.; Trecarichi, E.M.; Posteraro, P.; Losito, A.R.; De Luca, A.; Sanguinetti, M.; Fadda, G.; Cauda, R.; Posteraro, B. Risk Factors and Outcomes of Candidemia Caused by Biofilm-Forming Isolates in a Tertiary Care Hospital. PLoS ONE 2012, 7, e33705. [CrossRef]

20. Karpiński, T.M. Essential Oils of Lamiaceae Family Plants as Antifungals. Biomolecules 2020, 10, 103. [CrossRef]

21. Müller-Sepúlveda, A.; Chevecich, C.C.; Jara, J.A.; Belmar, C.; Sandoval, P.; Meyer, R.S.; Quijada, R.; Moura, S.; López-Muñoz, R.; Díaz-Dosque, M.; et al. Chemical Characterization of Lavandula Dentata Essential Oil Cultivated in Chile and Its Antibiofilm Effect against Candida Albicans. Planta Med. 2020, 86, 1225-1234. [CrossRef]

22. Motamedi, M.; Saharkhiz, M.J.; Pakshir, K.; Amini Akbarabadi, S.; Alikhani Khordshami, M.; Asadian, F.; Zareshahrabadi, Z.; Zomorodian, K. Chemical Compositions and Antifungal Activities of Satureja Macrosiphon against Candida and Aspergillus Species. Curr. Med. Mycol. 2019, 5, 20-25. [CrossRef]

23. Abu-Darwish, M.S.; Cabral, C.; Gonçalves, M.J.; Cavaleiro, C.; Cruz, M.T.; Paoli, M.; Tomi, F.; Efferth, T.; Salgueiro, L. Ziziphora Tenuior, L. Essential Oil from Dana Biosphere Reserve (Southern Jordan); Chemical Characterization and Assessment of Biological Activities. J. Ethnopharmacol. 2016, 194, 963-970. [CrossRef] [PubMed]

24. Abu-Darwish, M.S.; Cabral, C.; Gonçalves, M.J.; Cavaleiro, C.; Cruz, M.T.; Zulfiqar, A.; Khan, I.A.; Efferth, T.; Salgueiro, L. Chemical Composition and Biological Activities of Artemisia Judaica Essential Oil from Southern Desert of Jordan. J. Ethnopharmacol. 2016, 191, 161-168. [CrossRef]

25. Soliman, S.S.M.; Semreen, M.H.; El-Keblawy, A.A.; Abdullah, A.; Uppuluri, P.; Ibrahim, A.S. Assessment of Herbal Drugs for Promising Anti-Candida Activity. BMC Complement. Altern. Med. 2017, 17, 257. [CrossRef]

26. Jafri, H.; Ahmad, I. Thymus Vulgaris Essential Oil and Thymol Inhibit Biofilms and Interact Synergistically with Antifungal Drugs against Drug Resistant Strains of Candida Albicans and Candida Tropicalis. J. Mycol. Med. 2020, 30, 100911. [CrossRef] [PubMed]

27. Wang, Z.-J.; Zhu, Y.-Y.; Yi, X.; Zhou, Z.-S.; He, Y.-J.; Zhou, Y.; Qi, Z.-H.; Jin, D.-N.; Zhao, L.-X.; Luo, X.-D. Bioguided Isolation, Identification and Activity Evaluation of Antifungal Compounds from Acorus Tatarinowii Schott. J. Ethnopharmacol. 2020, 261, 113119. [CrossRef]

28. Said, M.M.; Watson, C.; Grando, D. Garlic Alters the Expression of Putative Virulence Factor Genes SIR2 and ECE1 in Vulvovaginal C. Albicans Isolates. Sci. Rep. 2020, 10, 3615. [CrossRef]

29. Bersan, S.M.F.; Galvão, L.C.C.; Goes, V.F.F.; Sartoratto, A.; Figueira, G.M.; Rehder, V.L.G.; Alencar, S.M.; Duarte, R.M.T.; Rosalen, P.L.; Duarte, M.C.T. Action of Essential Oils from Brazilian Native and Exotic Medicinal Species on Oral Biofilms. BMC Complement. Altern. Med. 2014, 14, 451. [CrossRef]

30. Teodoro, G.R.; Gontijo, A.V.L.; Salvador, M.J.; Tanaka, M.H.; Brighenti, F.L.; Delbem, A.C.B.; Delbem, Á.C.B.; Koga-Ito, C.Y. Effects of Acetone Fraction From Buchenavia Tomentosa Aqueous Extract and Gallic Acid on Candida Albicans Biofilms and Virulence Factors. Front. Microbiol. 2018, 9, 647. [CrossRef] [PubMed]

31. Barros Cota, B.; Batista Carneiro de Oliveira, D.; Carla Borges, T.; Cristina Catto, A.; Valverde Serafim, C.; Rogelis Aquiles Rodrigues, A.; Kohlhoff, M.; Leomar Zani, C.; Assunção Andrade, A. Antifungal Activity of Extracts and Purified Saponins from the Rhizomes of Chamaecostus Cuspidatus against Candida and Trichophyton Species. J. Appl. Microbiol. 2021, 130, 61-75. [CrossRef] [PubMed]

32. Wijesinghe, G.K.; Maia, F.C.; de Oliveira, T.R.; de Feiria, S.N.B.; Joia, F.; Barbosa, J.P.; Boni, G.C.; de Cássia Orlandi Sardi, J.; Rosalen, P.L.; Höfling, J.F. Effect of Cinnamomum Verum Leaf Essential Oil on Virulence Factors of Candida Species and Determination of the In-Vivo Toxicity with Galleria Mellonella Model. Mem. Inst. Oswaldo. Cruz. 2020, 115, e200349. [CrossRef]

33. Pedroso, R.D.S.; Balbino, B.L.; Andrade, G.; Dias, M.C.P.S.; Alvarenga, T.A.; Pedroso, R.C.N.; Pimenta, L.P.; Lucarini, R.; Pauletti, P.M.; Januário, A.H.; et al. In Vitro and In Vivo Anti-Candida Spp. Activity of Plant-Derived Products. Plants 2019, 8, 494. [CrossRef]

34. Andrade, G.; Orlando, H.C.S.; Scorzoni, L.; Pedroso, R.S.; Abrão, F.; Carvalho, M.T.M.; Veneziani, R.C.S.; Ambrósio, S.R.; Bastos, J.K.; Mendes-Giannini, M.J.S.; et al. Brazilian Copaifera Species: Antifungal Activity against Clinically Relevant Candida Species, Cellular Target, and In Vivo Toxicity. J. Fungi 2020, 6, 153. [CrossRef]

35. de Almeida Freires, I.; Murata, R.M.; Furletti, V.F.; Sartoratto, A.; de Alencar, S.M.; Figueira, G.M.; de Oliveira Rodrigues, J.A.; Duarte, M.C.T.; Rosalen, P.L. Coriandrum Sativum L. (Coriander) Essential Oil: Antifungal Activity and Mode of Action on Candida spp., and Molecular Targets Affected in Human Whole-Genome Expression. PLoS ONE 2014, 9, e99086. [CrossRef]

36. Manoharan, R.K.; Lee, J.-H.; Kim, Y.-G.; Kim, S.-I.; Lee, J. Inhibitory Effects of the Essential Oils $\alpha$-Longipinene and Linalool on Biofilm Formation and Hyphal Growth of Candida Albicans. Biofouling 2017, 33, 143-155. [CrossRef] [PubMed]

37. Khan, M.S.A.; Ahmad, I. Biofilm Inhibition by Cymbopogon Citratus and Syzygium Aromaticum Essential Oils in the Strains of Candida Albicans. J. Ethnopharmacol. 2012, 140, 416-423. [CrossRef] [PubMed]

38. Agarwal, V.; Lal, P.; Pruthi, V. Prevention of Candida Albicans Biofilm by Plant Oils. Mycopathologia 2008, 165, 13-19. [CrossRef] [PubMed]

39. De Toledo, L.G.; Ramos, M.A.D.S.; Spósito, L.; Castilho, E.M.; Pavan, F.R.; Lopes, É.D.O.; Zocolo, G.J.; Silva, F.A.N.; Soares, T.H.; Dos Santos, A.G.; et al. Essential Oil of Cymbopogon Nardus (L.) Rendle: A Strategy to Combat Fungal Infections Caused by Candida Species. Int. J. Mol. Sci. 2016, 17, 1252. [CrossRef]

40. Hendry, E.R.; Worthington, T.; Conway, B.R.; Lambert, P.A. Antimicrobial Efficacy of Eucalyptus Oil and 1,8-Cineole Alone and in Combination with Chlorhexidine Digluconate against Microorganisms Grown in Planktonic and Biofilm Cultures. J. Antimicrob. Chemother. 2009, 64, 1219-1225. [CrossRef] 
41. Quatrin, P.M.; Verdi, C.M.; de Souza, M.E.; de Godoi, S.N.; Klein, B.; Gundel, A.; Wagner, R.; de Almeida Vaucher, R.; Ourique, A.F.; Santos, R.C.V. Antimicrobial and Antibiofilm Activities of Nanoemulsions Containing Eucalyptus Globulus Oil against Pseudomonas Aeruginosa and Candida spp. Microb. Pathog. 2017, 112, 230-242. [CrossRef] [PubMed]

42. Sardi, J.d.C.O.; Freires, I.A.; Lazarini, J.G.; Infante, J.; de Alencar, S.M.; Rosalen, P.L. Unexplored Endemic Fruit Species from Brazil: Antibiofilm Properties, Insights into Mode of Action, and Systemic Toxicity of Four Eugenia spp. Microb. Pathog. 2017, 105, 280-287. [CrossRef]

43. Popović, V.; Stojković, D.; Nikolić, M.; Heyerick, A.; Petrović, S.; Soković, M.; Niketić, M. Extracts of Three Laserpitium L. Species and Their Principal Components Laserpitine and Sesquiterpene Lactones Inhibit Microbial Growth and Biofilm Formation by Oral Candida Isolates. Food. Funct. 2015, 6, 1205-1211. [CrossRef]

44. Benzaid, C.; Belmadani, A.; Djeribi, R.; Rouabhia, M. The Effects of Mentha $\times$ Piperita Essential Oil on C. Albicans Growth, Transition, Biofilm Formation, and the Expression of Secreted Aspartyl Proteinases Genes. Antibiotics 2019, 8, 10. [CrossRef] [PubMed]

45. Cannas, S.; Molicotti, P.; Usai, D.; Maxia, A.; Zanetti, S. Antifungal, Anti-Biofilm and Adhesion Activity of the Essential Oil of Myrtus Communis L. against Candida Species. Nat. Prod. Res. 2014, 28, 2173-2177. [CrossRef]

46. Stojković, D.; Dias, M.I.; Drakulić, D.; Barros, L.; Stevanović, M.; C F R Ferreira, I.; D Soković, M. Methanolic Extract of the Herb Ononis Spinosa L. Is an Antifungal Agent with No Cytotoxicity to Primary Human Cells. Pharmaceuticals 2020, 13, 78. [CrossRef] [PubMed]

47. Souza, C.M.C.; Pereira Junior, S.A.; Moraes, T.d.S.; Damasceno, J.L.; Amorim Mendes, S.; Dias, H.J.; Stefani, R.; Tavares, D.C.; Martins, C.H.G.; Crotti, A.E.M.; et al. Antifungal Activity of Plant-Derived Essential Oils on Candida Tropicalis Planktonic and Biofilms Cells. Med. Mycol. 2016, 54, 515-523. [CrossRef] [PubMed]

48. Curvelo, J.A.R.; Marques, A.M.; Barreto, A.L.S.; Romanos, M.T.V.; Portela, M.B.; Kaplan, M.A.C.; Soares, R.M.A. A Novel Nerolidol-Rich Essential Oil from Piper Claussenianum Modulates Candida Albicans Biofilm. J. Med. Microbiol. 2014, 63, 697-702. [CrossRef] [PubMed]

49. Bakkiyaraj, D.; Nandhini, J.R.; Malathy, B.; Pandian, S.K. The Anti-Biofilm Potential of Pomegranate (Punica Granatum L.) Extract against Human Bacterial and Fungal Pathogens. Biofouling 2013, 29, 929-937. [CrossRef] [PubMed]

50. Alves-Silva, J.M.; Zuzarte, M.; Gonçalves, M.J.; Cruz, M.T.; Cavaleiro, C.; Salgueiro, L. Unveiling the Bioactive Potential of the Essential Oil of a Portuguese Endemism, Santolina Impressa. J. Ethnopharmacol. 2019, 244, 112120. [CrossRef]

51. Sharifzadeh, A.; Khosravi, A.R.; Ahmadian, S. Chemical Composition and Antifungal Activity of Satureja Hortensis L. Essentiall Oil against Planktonic and Biofilm Growth of Candida Albicans Isolates from Buccal Lesions of HIV(+) Individuals. Microb. Pathog. 2016, 96, 1-9. [CrossRef]

52. Kipanga, P.N.; Liu, M.; Panda, S.K.; Mai, A.H.; Veryser, C.; Van Puyvelde, L.; De Borggraeve, W.M.; Van Dijck, P.; Matasyoh, J.; Luyten, W. Biofilm Inhibiting Properties of Compounds from the Leaves of Warburgia Ugandensis Sprague Subsp. Ugandensis against Candida and Staphylococcal Biofilms. J. Ethnopharmacol. 2020, 248, 112352. [CrossRef] [PubMed]

53. Gabriela, N.; Rosa, A.M.; Catiana, Z.I.; Soledad, C.; Mabel, O.R.; Esteban, S.J.; Veronica, B.; Daniel, W.; Ines, I.M. The Effect of Zuccagnia Punctata, an Argentine Medicinal Plant, on Virulence Factors from Candida Species. Nat. Prod. Commun. 2014, 9 , 933-936. [CrossRef]

54. Karpiński, T.M. Efficacy of Octenidine against Pseudomonas Aeruginosa Strains. Eur. J. Biol. Res. 2019, 9, 135-140.

55. Loures, F.V.; Levitz, S.M. XTT Assay of Antifungal Activity. Bio. Protoc. 2015, 5, e1543. [CrossRef] [PubMed]

56. Gonçalves, M.J.; Vicente, A.M.; Cavaleiro, C.; Salgueiro, L. Composition and Antifungal Activity of the Essential Oil of Mentha Cervina from Portugal. Nat. Prod. Res. 2007, 21, 867-871. [CrossRef]

57. Ćavar, S.; Vidic, D.; Maksimović, M. Volatile Constituents, Phenolic Compounds, and Antioxidant Activity of Calamintha Glandulosa (Req.) Bentham. J. Sci. Food Agric. 2013, 93, 1758-1764. [CrossRef]

58. Khan, M.S.A.; Ahmad, I. Antibiofilm Activity of Certain Phytocompounds and Their Synergy with Fluconazole against Candida Albicans Biofilms. J. Antimicrob. Chemother. 2012, 67, 618-621. [CrossRef]

59. Pemmaraju, S.C.; Pruthi, P.A.; Prasad, R.; Pruthi, V. Candida Albicans Biofilm Inhibition by Synergistic Action of Terpenes and Fluconazole. Indian J. Exp. Biol. 2013, 51, 1032-1037.

60. EUCAST: Breakpoints for Antifungals. Available online: https://eucast.org/astoffungi/clinicalbreakpointsforantifungals/ (accessed on 19 March 2021).

61. Messier, C.; Grenier, D. Effect of Licorice Compounds Licochalcone A, Glabridin and Glycyrrhizic Acid on Growth and Virulence Properties of Candida Albicans. Mycoses 2011, 54, e801-e806. [CrossRef] [PubMed]

62. Cao, Y.; Dai, B.; Wang, Y.; Huang, S.; Xu, Y.; Cao, Y.; Gao, P.; Zhu, Z.; Jiang, Y. In Vitro Activity of Baicalein against Candida Albicans Biofilms. Int. J. Antimicrob. Agents 2008, 32, 73-77. [CrossRef] [PubMed]

63. Cretton, S.; Dorsaz, S.; Azzollini, A.; Favre-Godal, Q.; Marcourt, L.; Ebrahimi, S.N.; Voinesco, F.; Michellod, E.; Sanglard, D.; Gindro, K.; et al. Antifungal Quinoline Alkaloids from Waltheria Indica. J. Nat. Prod. 2016, 79, 300-307. [CrossRef]

64. Cheng, A.; Sun, L.; Wu, X.; Lou, H. The Inhibitory Effect of a Macrocyclic Bisbibenzyl Riccardin D on the Biofilms of Candida Albicans. Biol. Pharm. Bull. 2009, 32, 1417-1421. [CrossRef] [PubMed]

65. Hu, D.-D.; Zhang, R.-L.; Zou, Y.; Zhong, H.; Zhang, E.-S.; Luo, X.; Wang, Y.; Jiang, Y.-Y. The Structure-Activity Relationship of Pterostilbene Against Candida Albicans Biofilms. Molecules 2017, 22, 360. [CrossRef] 
66. Feldman, M.; Sionov, R.V.; Mechoulam, R.; Steinberg, D. Anti-Biofilm Activity of Cannabidiol against Candida Albicans. Microorganisms 2021, 9, 441. [CrossRef] [PubMed]

67. Xie, C.; Sun, L.; Meng, L.; Wang, M.; Xu, J.; Bartlam, M.; Guo, Y. Sesquiterpenes from Carpesium Macrocephalum Inhibit Candida Albicans Biofilm Formation and Dimorphism. Bioorg. Med. Chem. Lett. 2015, 25, 5409-5411. [CrossRef] [PubMed]

68. Raut, J.S.; Shinde, R.B.; Chauhan, N.M.; Karuppayil, S.M. Phenylpropanoids of Plant Origin as Inhibitors of Biofilm Formation by Candida Albicans. J. Microbiol. Biotechnol. 2014, 24, 1216-1225. [CrossRef] [PubMed]

69. Raut, J.S.; Shinde, R.B.; Chauhan, N.M.; Karuppayil, S.M. Terpenoids of Plant Origin Inhibit Morphogenesis, Adhesion, and Biofilm Formation by Candida Albicans. Biofouling 2013, 29, 87-96. [CrossRef] [PubMed]

70. Ivanov, M.; Kannan, A.; Stojković, D.S.; Glamočlija, J.; Calhelha, R.C.; Ferreira, I.C.F.R.; Sanglard, D.; Soković, M. Camphor and Eucalyptol-Anticandidal Spectrum, Antivirulence Effect, Efflux Pumps Interference and Cytotoxicity. Int. J. Mol. Sci. 2021, 22, 483. [CrossRef] [PubMed]

71. Dalleau, S.; Cateau, E.; Bergès, T.; Berjeaud, J.-M.; Imbert, C. In Vitro Activity of Terpenes against Candida Biofilms. Int. J. Antimicrob. Agents 2008, 31, 572-576. [CrossRef] [PubMed]

72. Touil, H.F.Z.; Boucherit, K.; Boucherit-Otmani, Z.; Khoder, G.; Madkour, M.; Soliman, S.S.M. Optimum Inhibition of AmphotericinB-Resistant Candida Albicans Strain in Single- and Mixed-Species Biofilms by Candida and Non-Candida Terpenoids. Biomolecules 2020, 10, 342. [CrossRef]

73. Janeczko, M.; Masłyk, M.; Kubiński, K.; Golczyk, H. Emodin, a Natural Inhibitor of Protein Kinase CK2, Suppresses Growth, Hyphal Development, and Biofilm Formation of Candida Albicans. Yeast 2017, 34, 253-265. [CrossRef] [PubMed]

74. Ali, I.; Khan, F.G.; Suri, K.A.; Gupta, B.D.; Satti, N.K.; Dutt, P.; Afrin, F.; Qazi, G.N.; Khan, I.A. In Vitro Antifungal Activity of Hydroxychavicol Isolated from Piper Betle L. Ann. Clin. Microbiol. Antimicrob. 2010, 9, 7. [CrossRef] [PubMed]

75. Abirami, G.; Alexpandi, R.; Durgadevi, R.; Kannappan, A.; Veera Ravi, A. Inhibitory Effect of Morin Against Candida Albicans Pathogenicity and Virulence Factor Production: An in Vitro and in Vivo Approaches. Front. Microbiol. 2020, 11, 561298. [CrossRef] [PubMed]

76. Rivas da Silva, A.C.; Lopes, P.M.; Barros de Azevedo, M.M.; Costa, D.C.M.; Alviano, C.S.; Alviano, D.S. Biological Activities of $\alpha$-Pinene and $\beta$-Pinene Enantiomers. Molecules 2012, 17, 6305-6316. [CrossRef] [PubMed]

77. Lemos, A.S.O.; Florêncio, J.R.; Pinto, N.C.C.; Campos, L.M.; Silva, T.P.; Grazul, R.M.; Pinto, P.F.; Tavares, G.D.; Scio, E.; Apolônio, A.C.M.; et al. Antifungal Activity of the Natural Coumarin Scopoletin Against Planktonic Cells and Biofilms From a Multidrug-Resistant Candida Tropicalis Strain. Front. Microbiol. 2020, 11, 1525. [CrossRef]

78. Kim, H.-R.; Eom, Y.-B. Antifungal and Anti-Biofilm Effects of 6-Shogaol against Candida Auris. J. Appl. Microbiol. 2020. [CrossRef] [PubMed]

79. Dal Piaz, F.; Bader, A.; Malafronte, N.; D’Ambola, M.; Petrone, A.M.; Porta, A.; Ben Hadda, T.; De Tommasi, N.; Bisio, A.; Severino, L. Phytochemistry of Compounds Isolated from the Leaf-Surface Extract of Psiadia Punctulata (DC.) Vatke Growing in Saudi Arabia. Phytochemistry 2018, 155, 191-202. [CrossRef] [PubMed]

80. Shu, C.; Sun, L.; Zhang, W. Thymol Has Antifungal Activity against Candida Albicans during Infection and Maintains the Innate Immune Response Required for Function of the P38 MAPK Signaling Pathway in Caenorhabditis Elegans. Immunol. Res. 2016, 64, 1013-1024. [CrossRef] [PubMed]

81. Braga, P.C.; Culici, M.; Alfieri, M.; Dal Sasso, M. Thymol Inhibits Candida Albicans Biofilm Formation and Mature Biofilm. Int. J. Antimicrob. Agents 2008, 31, 472-477. [CrossRef]

82. Mandal, S.M.; Migliolo, L.; Franco, O.L.; Ghosh, A.K. Identification of an Antifungal Peptide from Trapa Natans Fruits with Inhibitory Effects on Candida Tropicalis Biofilm Formation. Peptides 2011, 32, 1741-1747. [CrossRef] [PubMed]

83. Patel, M.; Srivastava, V.; Ahmad, A. Dodonaea Viscosa Var Angustifolia Derived 5,6,8-Trihydroxy-7,4' Dimethoxy Flavone Inhibits Ergosterol Synthesis and the Production of Hyphae and Biofilm in Candida Albicans. J. Ethnopharmacol. 2020, $259,112965$. [CrossRef] 